\title{
Measurement of polymers with 3D optical scanners: evaluation of the subsurface scattering effect through five miniature step gauges
}

Guerra, M G; Gregersen, Søren K. S.; Frisvad, Jeppe Revall; De Chiffre, Leonardo; Lavecchia, F; Galantucci, L M

Published in:

Measurement Science and Technology

Link to article, DOI:

10.1088/1361-6501/ab3edb

Publication date:

2020

Document Version

Peer reviewed version

Link back to DTU Orbit

Citation $(A P A)$ :

Guerra, M. G., Gregersen, S. K. S., Frisvad, J. R., De Chiffre, L., Lavecchia, F., \& Galantucci, L. M. (2020). Measurement of polymers with 3D optical scanners: evaluation of the subsurface scattering effect through five miniature step gauges. Measurement Science and Technology, 31(1), [015010]. https://doi.org/10.1088/1361$6501 / \mathrm{ab3edb}$

\section{General rights}

Copyright and moral rights for the publications made accessible in the public portal are retained by the authors and/or other copyright owners and it is a condition of accessing publications that users recognise and abide by the legal requirements associated with these rights.

- Users may download and print one copy of any publication from the public portal for the purpose of private study or research.

- You may not further distribute the material or use it for any profit-making activity or commercial gain

- You may freely distribute the URL identifying the publication in the public portal 


\title{
Measurement of polymers with 3D optical scanners: evaluation of the subsurface scattering effect through five miniature step gauges
}

\author{
Guerra M.G.*, Gregersen S. S.**, Frisvad J. R.**, De Chiffre L.***, Lavecchia F.*, Galantucci L. M.* \\ *Department of Mechanics, Mathematics and Management, Polytechnic University of Bari, viale Japigia 182, 70126 Bari - Italy \\ **Department of Applied Mathematics and Computer Science, Technical University of Denmark, Richard Petersens Plads 321, DK-2800 Kgs. \\ Lyngby, Denmark \\ ***Department of Mechanical Engineering, Technical University of Denmark, Produktionstorvet 425, DK-2800 Kgs. Lyngby, Denmark
}

\begin{abstract}
In this work, the interaction between 3D optical scanners and objects realized with different materials and colours was investigated. The interaction is mainly related to the translucency of some materials, such as polymers, which is a category of materials ever more demanded in industry. At the same time, the increase of the geometrical complexity of manufactured parts raises the need for using 3D optical scanners thanks to their rapidity and effectiveness. Translucency causes a subsurface scattering effect that has been modelled for certain cases and affect the dimensional accuracy of measurements, as well as, the quality of reconstruction. Recently, an investigation considered its effect in dimensional verification using a structured light scanner with fringe projection. In this work, the investigation was extended to other 3D optical scanners exploiting different measuring principles: laser, structured light based on phase shifting and gray code, and photogrammetry. This was done in order to study how the subsurface scattering effect can vary with the materials, colours, and also the measuring principle used. To this end, five miniature step gauges made of different polymers and different colours were used, and the effects due to their different optical properties investigated. The analysis focused on dimensional verification of bidirectional lengths, first calibrated with a traceable CMM. The quality of the reconstruction was also evaluated through the flatness values.

According to the results obtained, translucency of materials has a dual and significant effect: a measurement bias and an increase of the uncertainty due to the different quality of reconstruction retrieved. Thus, it has to be considered properly, for each case.
\end{abstract}

Keywords: polymers, miniature step gauges, subsurface scattering, translucency, bidirectional lengths, 3D optical scanner, photogrammetry, laser scanner, structured light scanner.

\section{Introduction}

Optical based scanners offer many advantages. They are capable of acquiring a large amount of information in a short time and in a reliable way, which makes those instruments suitable for onmachine verification in manufacturing industry [1]. However, considerations regarding their accuracy and reliability are not generalizable. A certain number of error sources are known to affect optical based systems. Indeed, object material and colour can affect the accuracy of the systems in a way strictly dependent on the measuring principle adopted. 
Measuring polymers with 3D optical scanners became a widely spread measuring task in many industrial fields, due to the increase of polymers used in manufacturing, for both conventional manufacturing processes, such as injection moulding [2] and the newer additive technologies [3]. Polymers, indeed, exhibit optical properties, which interact with the optical measuring system depending on the specific material, the grade, the colour and the manufacturing process in terms of surface finish [4,5]. In particular, the optical interaction is due to transmission, scattering and absorption. Generally, the scattering phenomenon plays a major part in determining optical interaction, since polymers are translucent materials having microstructural features of dimensions comparable with the wavelength of visible light $(K=0.4-0.8 \mu \mathrm{m})$. When a 3D optical scanner is used for measuring a polymer, the measured point will not lie on the real external shape of the object, but it will be shifted a certain amount into the interior due to subsurface scattering. Several models exist that can be used for measuring the scattering properties of translucent materials [6-8]. The entity of the phenomenon is linked to the wavelength of the light source and to the optical properties of the material. Thus, it is of fundamental importance to know those parameters for each material, colour and surface finishing considered. However, specialized equipment is needed to measure optical properties [6-8]. In this work, the quantification of the subsurface scattering was carried out using a well known reference object: the step gauge. It was realized in different materials and colours and their bidirectional lengths analysed and compared with respect to CMM values. Bidirectional lengths take into account the "probing effect", which would represent, for non-contact measuring systems, the interaction between the measuring instrument and the optical properties of the workpiece.

\section{Research background}

The interest towards the optical properties of different materials, manufactured with different surface finishes, and, having different colours, is a topic of great interest, but it is not yet fully explored. National institutes for metrology developed some facilities for the characterization of several aspects affecting 3D optical instruments, and, in particular, how the materials and surface finish can affect the results in terms of number of acquired points, using specifically designed artefacts [9]. The influence of colour is investigated for a laser line scanner [10] and a commercial structured light scanner [11], considering a specific coded pattern. Both these researches use the x-rite ColorChecker chart [12], manufactured to provide a standard set of colour patches with known spectral reflectance for the photographic industry. Results confirm the expectations, and the surface colour is a factor to consider when dealing with optical-based instruments. Although, in that research, the effect of surface colours and materials on dimensional accuracy is not evaluated.

Generally, the difficulties related to digitization of translucent surfaces are overcome through different methods, such as bias correction, polarization difference imaging, modulated phase shifting, as well as matte coating of such surfaces [13]. The latter is employed to reach Lambertian conditions. However, a coating can change the overall size and the geometry of the sample, and it should always be checked with a profilometer. Moreover, it is difficult to achieve a uniform coating thickness especially if the geometrical complexity increases, and it is strongly related to operator skills and experience. This practise is widely used for large parts, where addition of a few micrometres of coating, even if not applied uniformly, does not significantly affect the accuracy and the total uncertainty [14].

Other approaches have, therefore, been developed to reduce the effects caused by subsurface scattering, and to improve the signal-to-noise ratio for structured light scanners. In particular, Chen et al. [15] developed a technique that combines polarization difference imaging (PDI) with structured light 3D scanning based on phase-shifting patterns to filter out subsurface scattering. PDI is an efficient tool for deblurring the phase-shifting pattern and obtaining a more robust 3D scanning of translucent materials. However, the depolarization length, that is, the distance that light needs to travel through the material before being unpolarized, is 3 to 10 mean free paths (depending on the scattering anisotropy of the material) for common translucent materials [16]. This means that while scanning may be more robust 
with this technique, it does not remove the bias problem (offset due to subsurface scattering). In a follow-up work, Chen et al. [17] obtained even better robustness when scanning translucent materials if using modulated phase-shifting, where a one-dimensional phase-shifting pattern is multiplied by a second shifted high-frequency pattern in the orthogonal direction. However, the focus of this work is scanning robustness rather than accuracy.

In a research by Gupta et al. $[18,19]$, new binary code patterns based on logical operations like exclusiveor (xor) are introduced to obtain more robust 3D scanning of scenes with global illumination effects (such as subsurface scattering). Again, the focus in this work is on obtaining a scan rather than on the dimensional accuracy of the scanned surface. Similarly, Kobayashi et al. [20] propose a complementary Gray code projection to improve 3D scanning based on structured light. These authors obtain more complete scans (better robustness) than the COMET5 scanner they are comparing with, but work with objects for which the true 3D shape is unknown. Thus, they too cannot investigate accuracy. Another technique for better robustness in scanning of translucent (or specular) materials is based on estimation of a homogeneous light transport matrix [21]. Many authors investigate robustness of optical 3D scanners encountering translucent objects, but few consider the bias problem, which affects the accuracy of the scans. To counteract the bias problem, Inoshita et al. [22] use a single scattering model to estimate both scattering properties and object shape. While their technique shows some potential, its accuracy suffers from low intensity of the singly scattered light.

In work by Godin et al. [23], a laser based 3D scanning system is used for digitizing marble sculptures. Marble's translucency and heterogeneous structure produce significant bias and increased noise in dimensional measurements. A bias was indeed observed, but, because of the strong surface reflection compared to the subsurface scattering, its impact is shown to be limited.

Wilm et al. [24] investigate on the accuracy of a structured light scanner using the same step gauges considered in the present work. This is accompanied by a dimensional verification and an uncertainty assessment. Results put in evidence a great influence of the material and the colours of the step gauges, but the experiments were limited to a single 3D optical scanner. Generally, when dealing with the subsurface scattering effect, one would consider active optically based systems [25]. Even so, the influence of the object material and colours is also of great importance for passive optical scanners, such as ones exploiting the photogrammetric principle. This is the case, since the success of the reconstruction is strictly connected to the visible surface texture, which determines the number of common features recognized among multi-view images of the object [26]. In previous work [4], a photogrammetric system is used for the measurement of microfluidic devices realized by additive manufacturing of polymers. Here, many criticalities arose due to the smoothness of those materials.

In this work, using the step gauges also used by Wilm et al. [24], the analysis is extended to other 3D optical scanners exploiting different measuring principles, in order to understand and quantify the interaction between the optical properties of each material with the measuring system adopted, in terms of measurement error and quality of reconstruction.

The paper is structured with a section dedicated to the Materials and Methods, describing the reference object, the measuring equipment, the measuring procedure and the method for the uncertainty assessment. Then, results will be reported in the dedicated section, followed by their discussion.

\section{Materials and methods}

In the following sections, a description of the miniature step gauge, the measuring equipment used and the measuring procedure are reported, together with a description of the method for the uncertainty assessment. 


\subsection{Step gauges description}

The step gauge was firstly adopted in [27] for the optical scanning of small parts, and it was fabricated with a bisacryl material. Afterwards, step gauges were used for the performance verification and systematic error correction of CT scanning systems. Different materials were used for the fabrication and mostly polymers due to their low density, which makes them suitable for CT scanning. According to [28] the polymers used, Peek and PPS with $40 \%$ of glass, resulted in step gauges fulfilling the needs for stability over time and surface quality. In this work, five step gauges having the same nominal dimensions, 58x7x8 $\mathrm{mm}^{3}$, but made of different polymers and colours [24], see Table 1, were scanned and measured using 3D optical scanners exploiting different measuring principles.

Table 1 List of the step gauges used in this work: materials and colours.

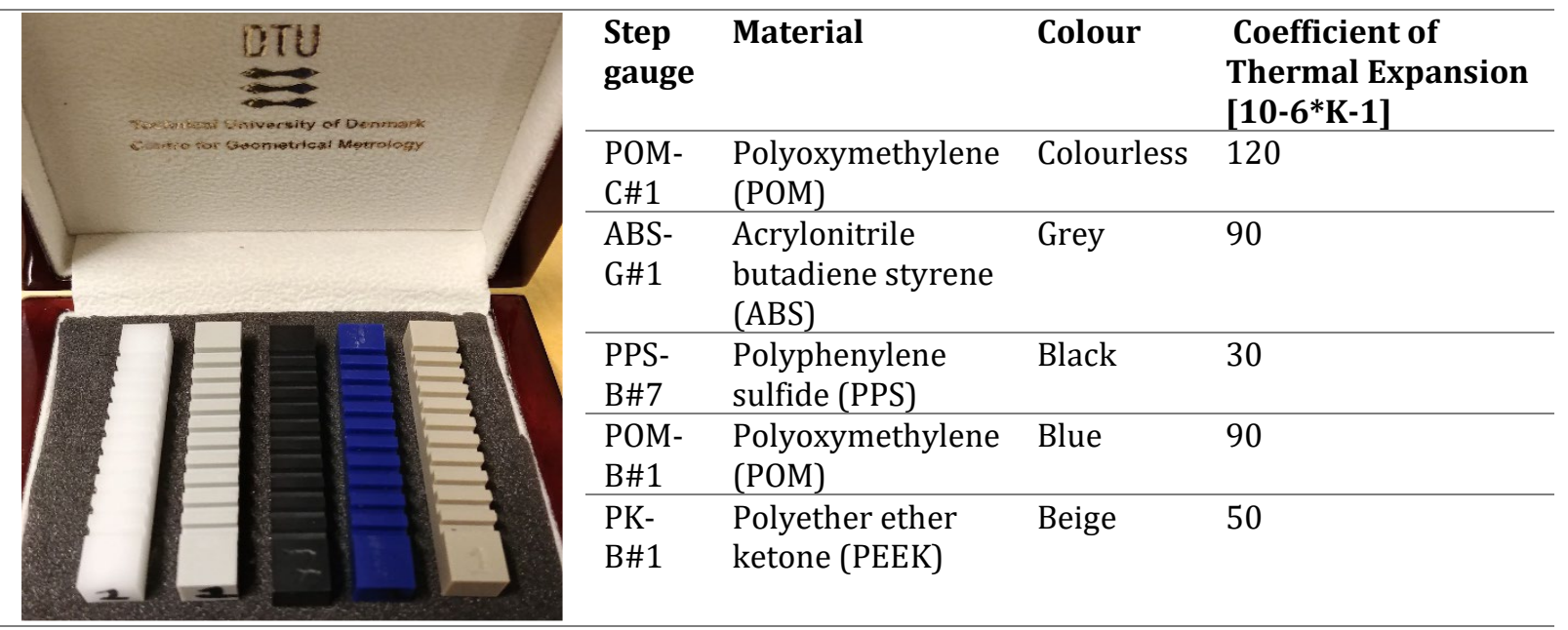

The step gauge geometry features unidirectional and bidirectional lengths. Their graphical definition is shown in Figure 1. The following equations serve to explain the effect of shifting the measuring point from the real surface towards the inside. Note that it is not possible to detect this effect with the unidirectional lengths $\left(L_{\mathrm{Um}}, L_{\mathrm{Uc}}\right)$, but it becomes possible with the bidirectional lengths $\left(L_{\mathrm{Bm}}, L_{\mathrm{Bc}}\right)$, where the "offset", expressed as $\Delta l$ in the equations, is added to the result twice.

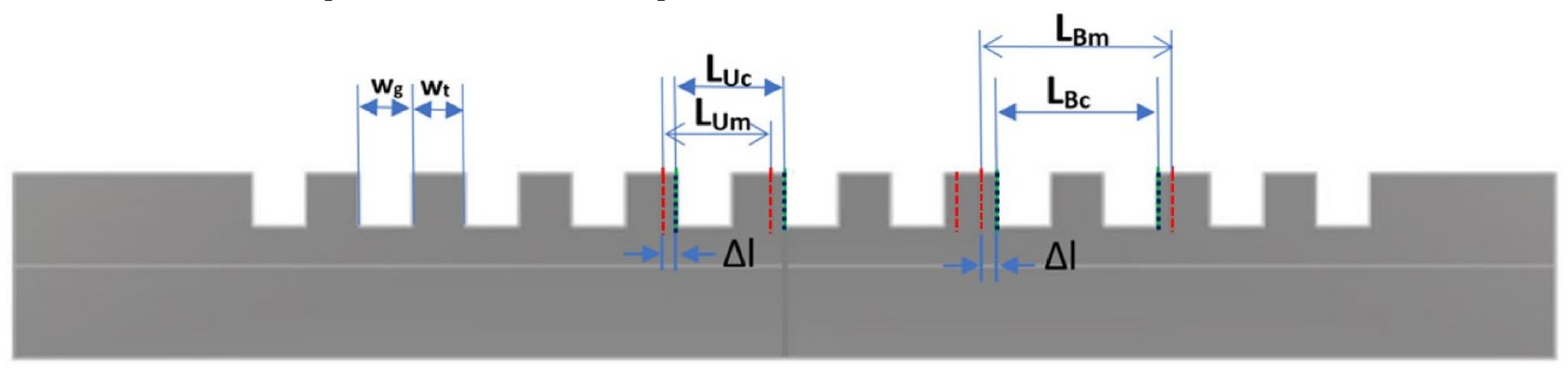

Figure 1 Step gauge unidirectional and bidirectional lengths definition.

$$
\begin{gathered}
L_{\mathrm{Um}}=w_{g}+w_{t}-\Delta l+\Delta l \\
L_{\mathrm{Bm}}=w_{g}+w_{t}+w_{g}+\Delta l+\Delta l,
\end{gathered}
$$

where

$L_{\mathrm{Bm}}$ is the measured bidirectional length;

$L_{\mathrm{Bc}}$ is the calibrated bidirectional length with a contact probe; 
$L_{\mathrm{Um}}$ is the measured unidirectional length;

$L_{\mathrm{Uc}}$ is the calibrated unidirectional length with a contact probe;

$w_{g}$ is the groove width;

$w_{t}$ is the tooth width;

$\Delta l$ is the offset respect to the real surface of the object;

In this work, the lengths reported in Figure 2, were considered as measurands.
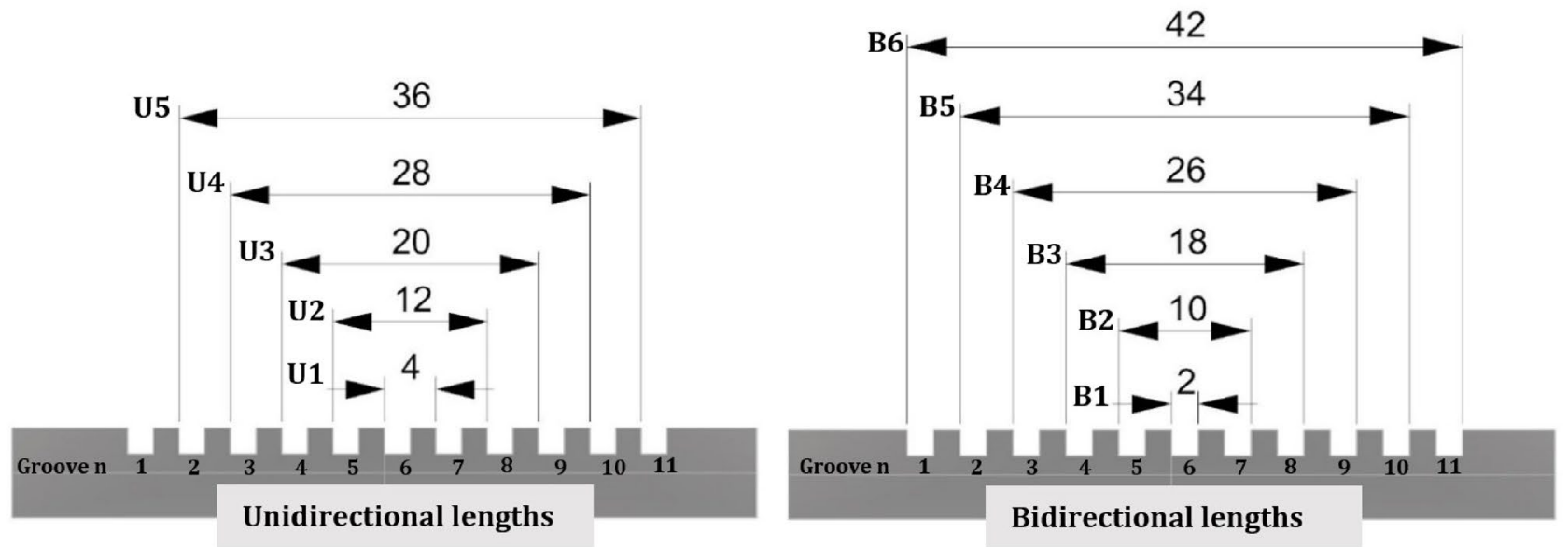

Figure 2 Unidirectional and bidirectional lengths considered. Measures are expressed in mm.

\subsection{D scanners used in this investigation}

The five step gauges were scanned with different optical instruments. A laser line scanner, two commercial structured light scanners, a structured light scanner realized at the Department of Applied Mathematics and Computer Science of the Technical University of Denmark and, finally, a photogrammetric scanner realized at the Department of Mechanics, Mathematics and Management of Polytechnic University of Bari. Both structured light scanners and the laser line scanner are counted among active optical systems, since a sinusoidal pattern or a laser strip is projected onto the object surface and then acquired, while, the photogrammetric system adopted is a passive system, without the projection of any pattern. The scanners are listed below and the scanning strategy reported for each case.

- 3SHAPE D800, a laser line scanner with a red beam $(\Lambda=630-680 \mathrm{~nm})$ with a resolution of 0.02 $\mathrm{mm}$. The scanner movements are computed by a tilting and rotating table, which allow the exploitation of different scanning strategies to scan complex and freeform parts. The scanning strategy adopted in this case was a tilt angle of $45^{\circ}$ and a rotary stage of $36^{\circ}$, which means 10 acquisitions. This scanner is recognized in the paper as LLS. Convince software was used for the data processing and the meshing operation.

- GOM ATOS III scan Rev. 2, equipped with 90 mm lenses, which allow measurement of a working volume of $60 \times 45 \times 30 \mathrm{~mm}^{3}$ with a resolution of $0.017 \mathrm{~mm}$. This is a structured light scanner with blue light, in order to avoid the influence of the environmental illumination. The scanning strategy adopted was a polar scan, with the step gauge positioned at the centre of rotary table and 8 acquisitions were carried out every $45^{\circ}$, while the sensor was tilted $45^{\circ}$ with respect to the rotary table (xy plane). This scanner is recognized in the paper as SLS-1. GOM Inspect software was used for point cloud processing and the meshing process.

- ATOS Capsule, an optical precision measuring machine (OPMM) for full-field digitizing of contoured part geometries. The system exploits the triple scan technology, with two cameras 
and the projection unit: precise fringe patterns are projected onto the surface of the object and are recorded by two cameras, based on the stereo camera principle. The system used in this work is a $12 \mathrm{MP}$ sensor with a measurement area of $120 \mathrm{~mm} \times 80 \mathrm{~mm}$, with a resolution of 0.027 $\mathrm{mm}$. The acquisitions were performed firstly with the sensor tilted at $90^{\circ}$ and then with the sensor tilted of $60^{\circ}$ while the turning table was rotated every $45^{\circ}$, which means 8 acquisitions. This scanner will be indicated in the next sections as SLS-2. GOM Inspect software was used for point cloud processing and the meshing process.

- The structured light scanner from DTU compute is set up according to Figure 3. It consists of two industrial cameras (Point Grey GS3-U3-91S6C-C, 9MPX) with $54 \mathrm{~mm}$ lenses and a field of view of approximately $250 \times 150 \times 150 \mathrm{~mm}$. It is also equipped with a pattern projector (LG PF1500, 1920x1080) and rotation stage (Newmark Systems RM- 5). It is equipped with a "white light" home cinema projector as light source. The two-frequency heterodyne principle was used for phase shift encoding with 40/41 fringe periods and 16/8 primary and secondary steps. Additionally, we also used a 8-step Gray code pattern including its binary inverse. A precision study has previously been performed on the scanner, see [29] for more technical details. Using the same method presented in [24], the step gauges were placed in a custom 3D printed fixture and scanned from 16 different angles. Alignment was based on a calibrated rotation axis finetuned using optical markers with an iterative marker-estimation algorithm. Reconstruction was done using Meshlab. These will be indicated in the next sections as SLS-PS and SLS-GC, according to the used approach: phase shift or gray code, respectively.

- PSSRT, a Photogrammetric Scanning System with Rotary Table, equipped with a Canon Eos 760D, a Canon EF $50 \mathrm{~mm}$ 1:1:8 II objective lens and an extension tube of $20 \mathrm{~mm}$, which determines a consequent ground resolution of $0.012 \mathrm{~mm} /$ pixel. The sensor was tilted of $45^{\circ}$ and 72 pictures were taken rotating the object with a step of $5^{\circ}$. The optimal light conditions were assured by using three led lamps, one placed at the top of the object and two placed behind the camera and inclined at the same tilt angle. The system shows characteristics comparable with the commercially available 3D optical scanners [30]. In this case, the adjustment of the scaling process was carried out through the calibrated unidirectional lengths. The reconstruction was carried out with Agisoft Photoscan v. 1.2.6.

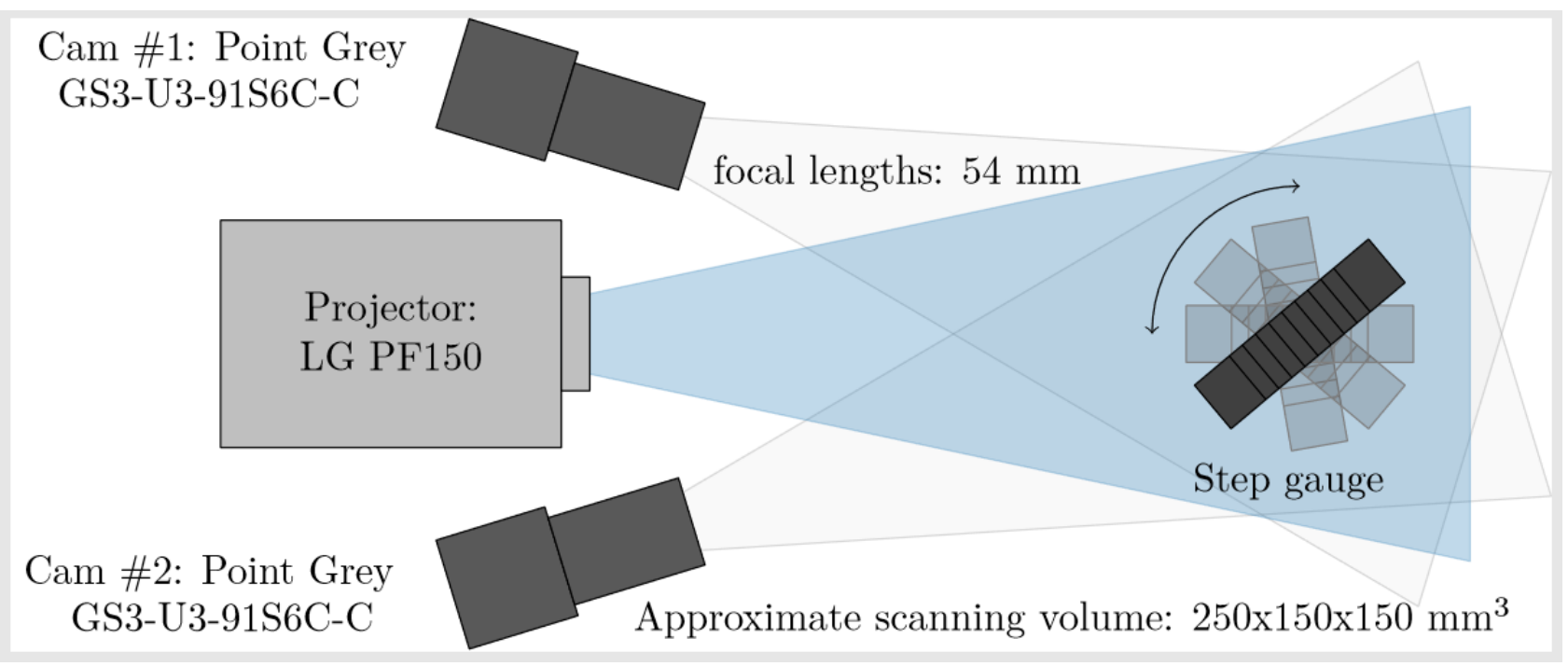

Figure 3 Structured light scanner from DTU Compute.

The step gauges were scanned five times for each instrument involved in the analysis. The acquired surfaces, in the form of polygonised meshes, were analysed with the GOM inspect software, and the distances between the groove sides were computed as distances between corresponding points on each groove side. Before computing the resulting errors, temperature compensation must be done for the LLS, the SLS-GC and the SLS-PS. Indeed, temperatures registered was $23.5^{\circ}$ for the LLS and 23.75 for the 
SLS-GC and SLS-PS. Humidity was also registered and it was ranging within $48-50 \%$ for the SLS, while it was not detectable for the other scanners, thus, a humidity compensation was not carried out in this investigation.

\subsection{Uncertainty assessment}

The uncertainty evaluation was conducted according to the ISO 14253-2 [31], as a simplification of the GUM approach [32].

The general equation is reported (Eq. 1.3) and the confidence level is set to $95 \%$ to which corresponds a coverage factor $\mathrm{k}=2$.

$U=k \sqrt{u_{r}^{2}+u_{w}^{2}+u_{e}^{2}+u_{p}^{2}}$

The uncertainty contributors are reported in Table 2. Starting from that, the uncertainty of calibration was considered as the uncertainty of calibration of each measurand (step gauge unidirectional and bidirectional lengths). The uncertainty component related to the instrument was not considered due to the difficult estimation of the MPE (Maximum Permissible Error) for the optical scanners considered. This is the case, since it is strongly dependent on the visible texture characteristics coming from the combination between materials and colours, while other approaches, e.g. considering the optical resolution, would have led to a high overestimation of the uncertainty. The uncertainty coming from the workpiece was considered as the standard deviation of the fitting error, which is the variability of the normal distances between each point on the real surface and the corresponding one on the fitted plane. The component due to the environmental temperature was also considered, and, finally, the uncertainty due to the measuring repeatability was evaluated as standard deviation of the mean of five repetitions.

Table 2 Uncertainty budget composition.

\begin{tabular}{|c|c|c|c|c|c|c|c|c|c|}
\hline $\begin{array}{l}\text { Uncertainty } \\
\text { component }\end{array}$ & Symbol & Type & Estimation & Distrib. & LLS & SLS-1 & SLS-2 & $\begin{array}{l}\text { SLS- } \\
\text { GC/PS }\end{array}$ & PPSRT \\
\hline Reference & $u_{r}$ & $B$ & $\begin{array}{l}\text { Reference } \\
\text { Uncertainty }\end{array}$ & Rect. & $\mathrm{Ui} / \sqrt{3}$ & $\mathrm{Ui} / \sqrt{3}$ & $\mathrm{Ui} / \sqrt{3}$ & $\mathrm{Ui} / \sqrt{3}$ & $\mathrm{Ui} / \sqrt{3}$ \\
\hline Workpiece & $u_{w}$ & $A$ & Form error & Rect. & $\begin{array}{c}\boldsymbol{\sigma} \text { fitting } \\
\text { error }\end{array}$ & $\begin{array}{c}\boldsymbol{\sigma} \text { fitting } \\
\text { error }\end{array}$ & $\begin{array}{c}\boldsymbol{\sigma} \text { fitting } \\
\text { error }\end{array}$ & $\begin{array}{c}\boldsymbol{\sigma} \text { fitting } \\
\text { error }\end{array}$ & $\begin{array}{c}\boldsymbol{\sigma} \text { fitting } \\
\text { error }\end{array}$ \\
\hline Environment & $u_{e}$ & B & $\begin{array}{l}\text { Temperature } \\
\text { variation }\end{array}$ & $\begin{array}{l}\text { U- } \\
\text { shaped }\end{array}$ & $\pm 1.5^{\circ} \mathrm{C}$ & $\pm 1^{\circ} \mathrm{C}$ & $\pm 1^{\circ} \mathrm{C}$ & $\pm 1.25^{\circ} \mathrm{C}$ & $\pm 1 C$ \\
\hline Repeatability & $u_{p}$ & $A$ & $\begin{array}{l}\text { Repeated } \\
\text { measurements }\end{array}$ & Normal & $\sigma_{\mathrm{LLS}} \sqrt{n}$ & $\sigma_{\mathrm{SLS}-1 / \sqrt{n}}$ & $\sigma_{\mathrm{SLS}-2} \sqrt{n}$ & $\sigma_{S L S} \sqrt{n}$ & $\sigma_{\mathrm{PSSRT}} \sqrt{n}$ \\
\hline
\end{tabular}

\section{Results}

Before reporting the overall numerical results, a summary of results is shown in Table 3. Results are divided into two categories: cooperative and not cooperative. Cooperative means that the point cloud acquisition produced analysable results, and, in that case, an indication of the errors registered was reported, while the not cooperative means that the measurement was not successful because of few points acquired on the sample surface, which made the reconstructed surfaces not analysable. In that case, in the table the text "No data" is reported.

Errors reported in Table 3 are defined as the maximum values of error registered for each combination scanner-object, considering the unidirectional lengths. 
Table 3 First summary of results of the step gauges acquisition. Errors reported are the maximum values of error, considering, for each case, the unidirectional lengths.

\begin{tabular}{|l|l|l|l|l|l|l|}
\hline & LLS & SLS-1 & SLS-2 & SLS-GC & SLS-PS & PSSRT \\
\hline POM-C\#1 & No data & No data & No data & No data & $0.03 \mathrm{~mm}$ & No data \\
\hline ABS-G\#1 & $0.005 \mathrm{~mm}$ & $0.002 \mathrm{~mm}$ & $0.015 \mathrm{~mm}$ & $0.05 \mathrm{~mm}$ & $0.005 \mathrm{~mm}$ & No data \\
\hline PPS-B\#7 & $0.003 \mathrm{~mm}$ & $0.001 \mathrm{~mm}$ & $0.001 \mathrm{~mm}$ & $0.03 \mathrm{~mm}$ & $0.015 \mathrm{~mm}$ & $0.002 \mathrm{~mm}$ \\
\hline POM-B\#1 & $0.03 \mathrm{~mm}$ & No data & No data & $0.04 \mathrm{~mm}$ & $0.01 \mathrm{~mm}$ & No data \\
\hline PK-B\#1 & $0.008 \mathrm{~mm}$ & $0.005 \mathrm{~mm}$ & $0.004 \mathrm{~mm}$ & $0.035 \mathrm{~mm}$ & $0.005 \mathrm{~mm}$ & No data \\
\hline & & & & & & \\
\hline
\end{tabular}

\subsection{Dimensional verification of the step gauge}

Firstly, unidirectional lengths were computed for each step gauge and each optical instrument that produced analysable results. The output variable chosen was the error evaluated with respect to the CMM calibrated values, following

$$
\text { Error }=\overline{X_{l \jmath}}-\overline{X_{\text {cal }}},
$$

where,

$\overline{X_{l]}}$ is the average value (five repetitions) of the analysed length belonging to the $i^{\text {th }}$ step gauge, measured with the $\mathrm{j}^{\text {th }}$ optical scanner and compared with $\overline{X_{\text {cal }}}$, the calibrated value for that length.

Considering the step gauge made of ABS, see Figure 4, the errors were comprised between $-0.05 \mathrm{~mm}$ and $0.01 \mathrm{~mm}$ for all the scanners involved. However, if only the LLS, SLS-1/2 are considered, errors registered were within $\pm 0.005 \mathrm{~mm}$, highlighting a good agreement among them. Uncertainties were assessed to be below $0.03 \mathrm{~mm}$. 


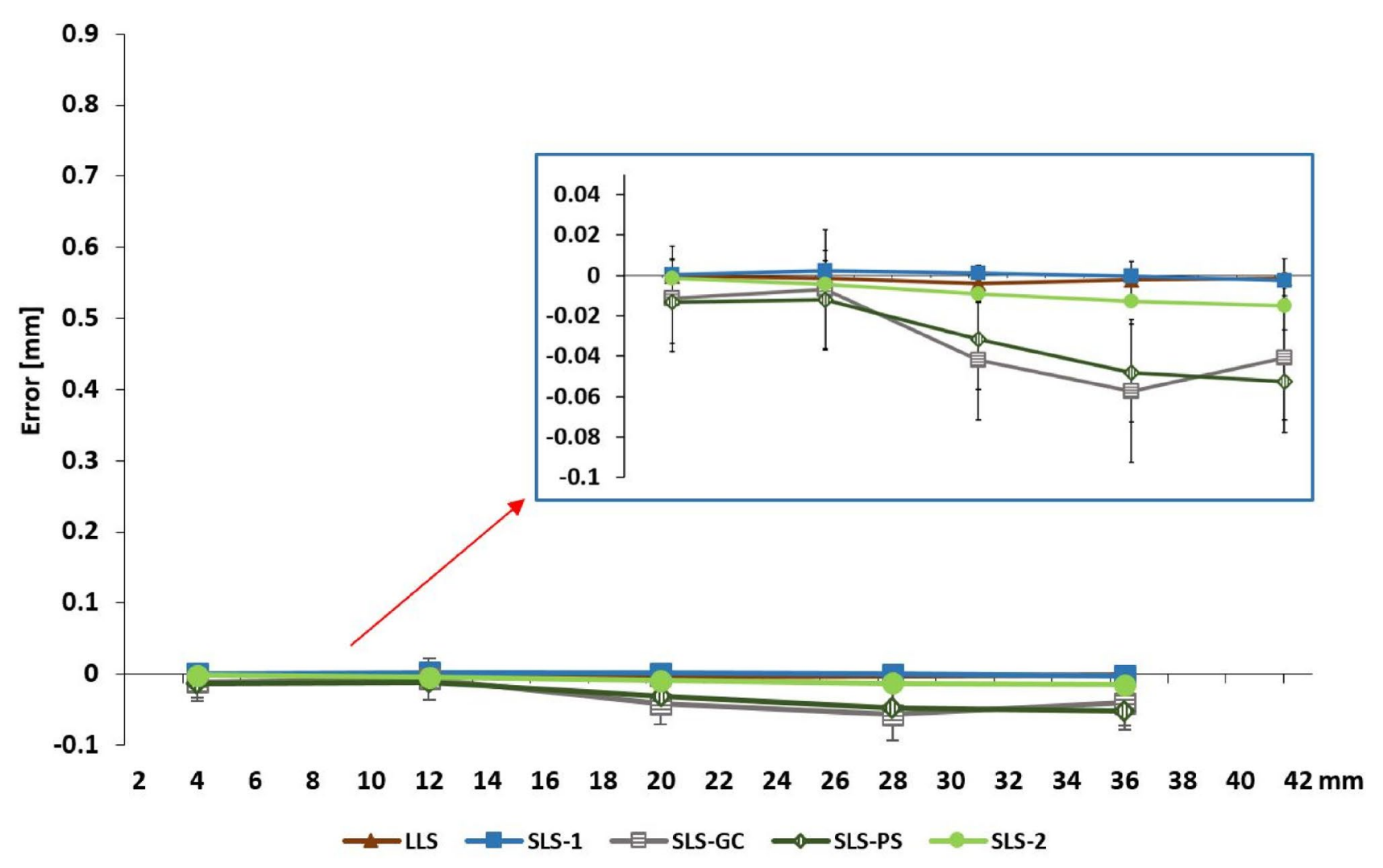

Figure 4 Errors on unidirectional lengths for the step gauge ABS-G\#1.

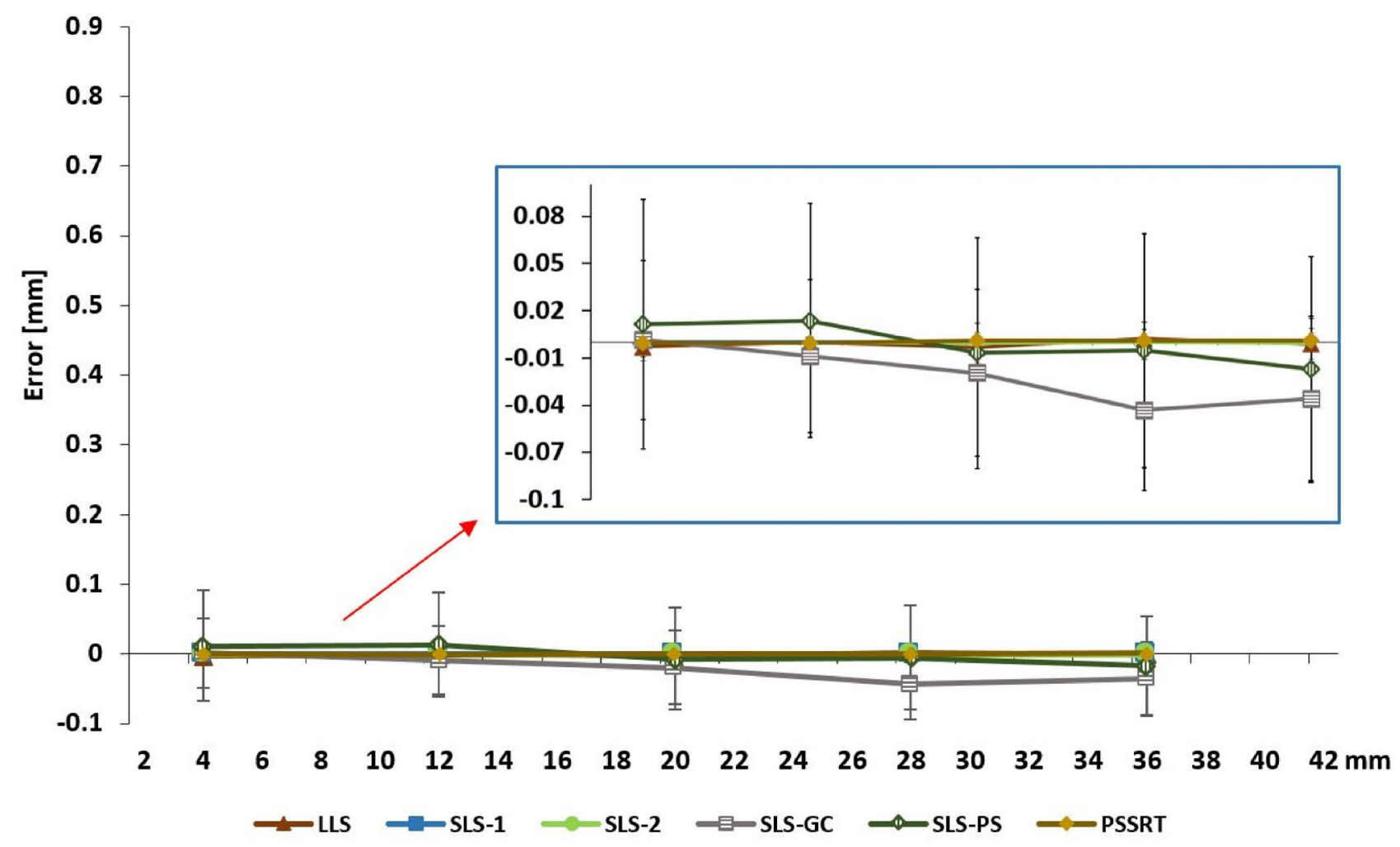

Figure 5 Errors on unidirectional lengths for the step gauge PPS-B\#7.

Same considerations could be done for the PPS-B\#7 step gauge, see Figure 5, where the group composed by LLS SLS- $1 / 2$ and SLS-PS showed errors comprised between \pm 0.02 . The SLS-GC showed instead errors 
up to $-0.04 \mathrm{~mm}$. Uncertainties were less than $0.01 \mathrm{~mm}$ for most of the scanners and $0.05 \mathrm{~mm}$ for SLS-GC and 0.06 for SLS-PS.

The PK-B\#1, see Figure 6, registered errors comprised between $\pm 0.005 \mathrm{~mm}$ for LLS, SLS-1/2 and errors within 0.02 and $-0.04 \mathrm{~mm}$ for the SLS-PS and SLS-GC. Uncertainties were about $0.005 \mathrm{~mm}$ for the SLS-1, $0.01 \mathrm{~mm}$ for LLS and SLS-2, and less than $0.02 \mathrm{~mm}$ for the rest of the scanners.

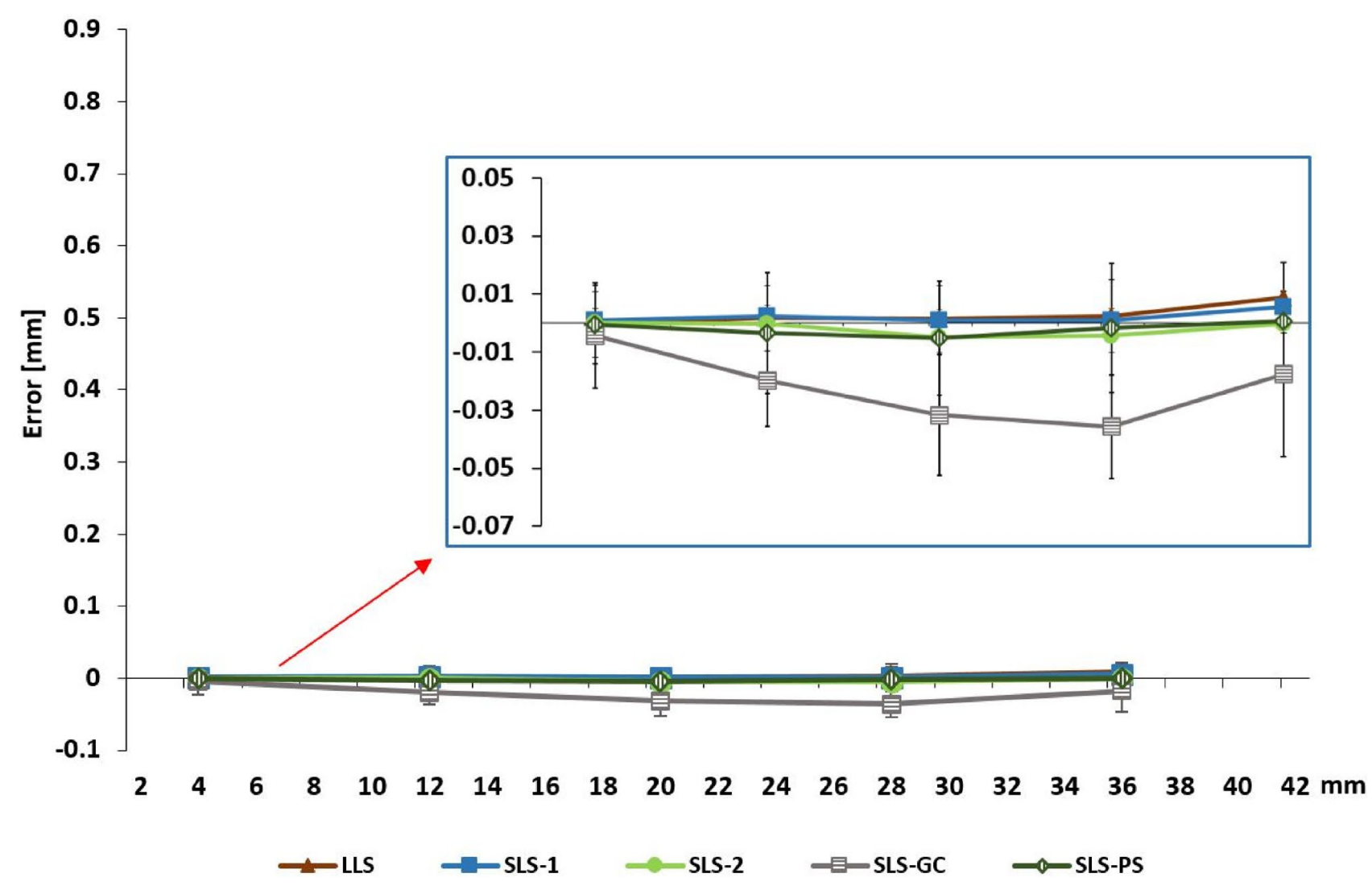

Figure 6 Errors on unidirectional lengths for the step gauge PK-B\#1.

The step gauge made of blue POM, see Figure 7, was acquired only by three scanners: the LLS, the SLSPS and SLS-GC. Errors registered for the LLS were the highest registered for this scanner and they were up to $-0.03 \mathrm{~mm}$. The SLS-PS and SLS-GC showed similar results. A relevant difference as compared with the other cases was found in the uncertainty. This was on the order of $0.05 \mathrm{~mm}$ for the SLS-PS and 0.04 for SLS-GC, while it was about $0.02 \mathrm{~mm}$ for the LLS. Finally, the step gauge made of colourless POM was analysed, it is reported in Figure 7, together with the blue POM. It was successfully acquired only by the SLS-PS, with errors and uncertainties comparable with the ones obtained for the POM-B\#1. 


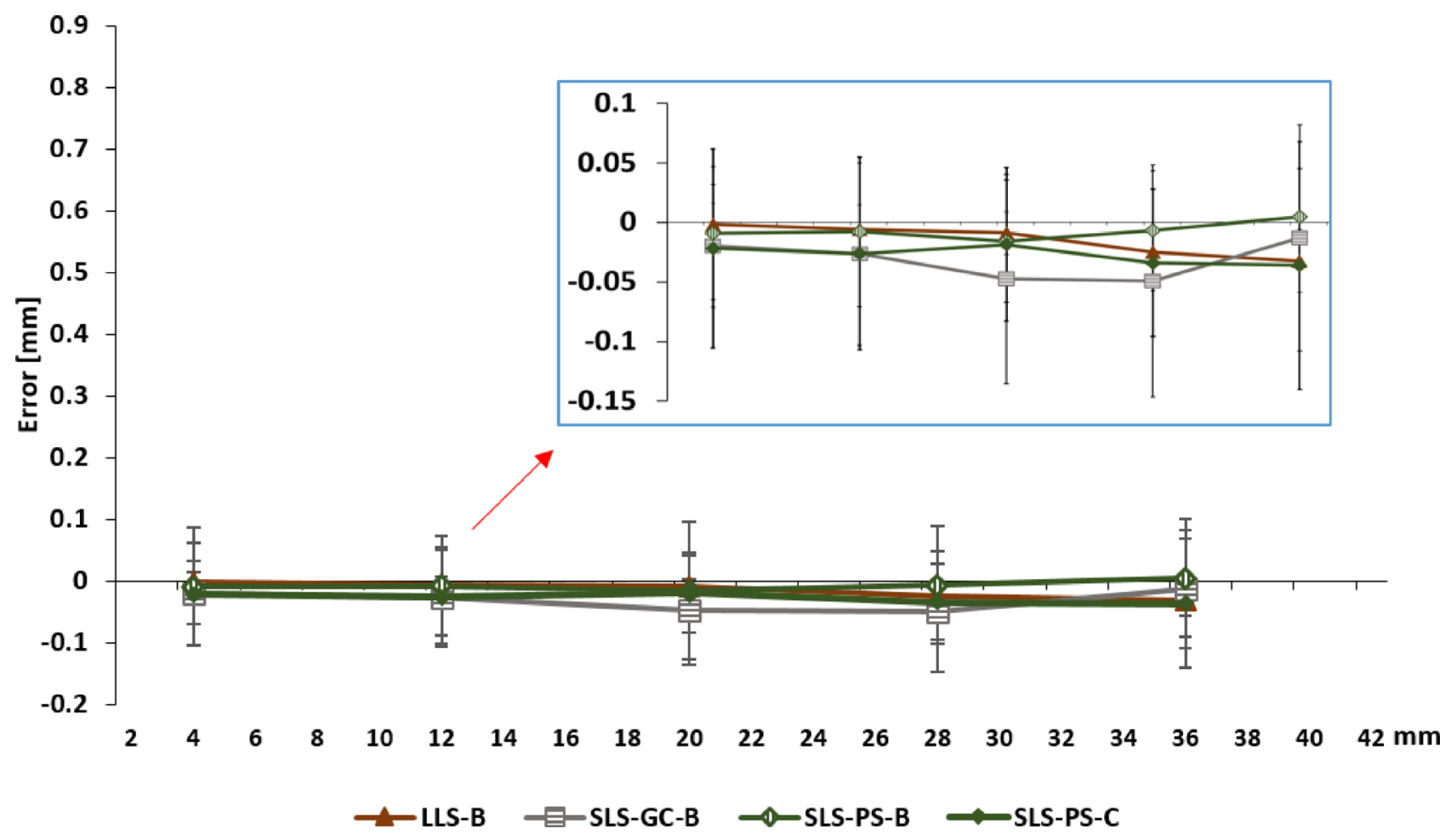

Figure 7 Errors on unidirectional lengths for the step gauges made of POM. The letter B, after the scanner name indicates the POM-B\#1 step gauge, while the letter $C$ indicates the POM-C\#1.

The general observation is that, regarding the unidirectional lengths, there are no relevant differences between the scanners involved in the investigation and, even in the case of SLS-GC and SLS-PS they were comprised in a narrow range. More important for the current investigation are the errors evaluated on bidirectional lengths, as explained in the introduction. As expected, errors evaluated on bidirectional lengths were higher than errors evaluated on unidirectional lengths.

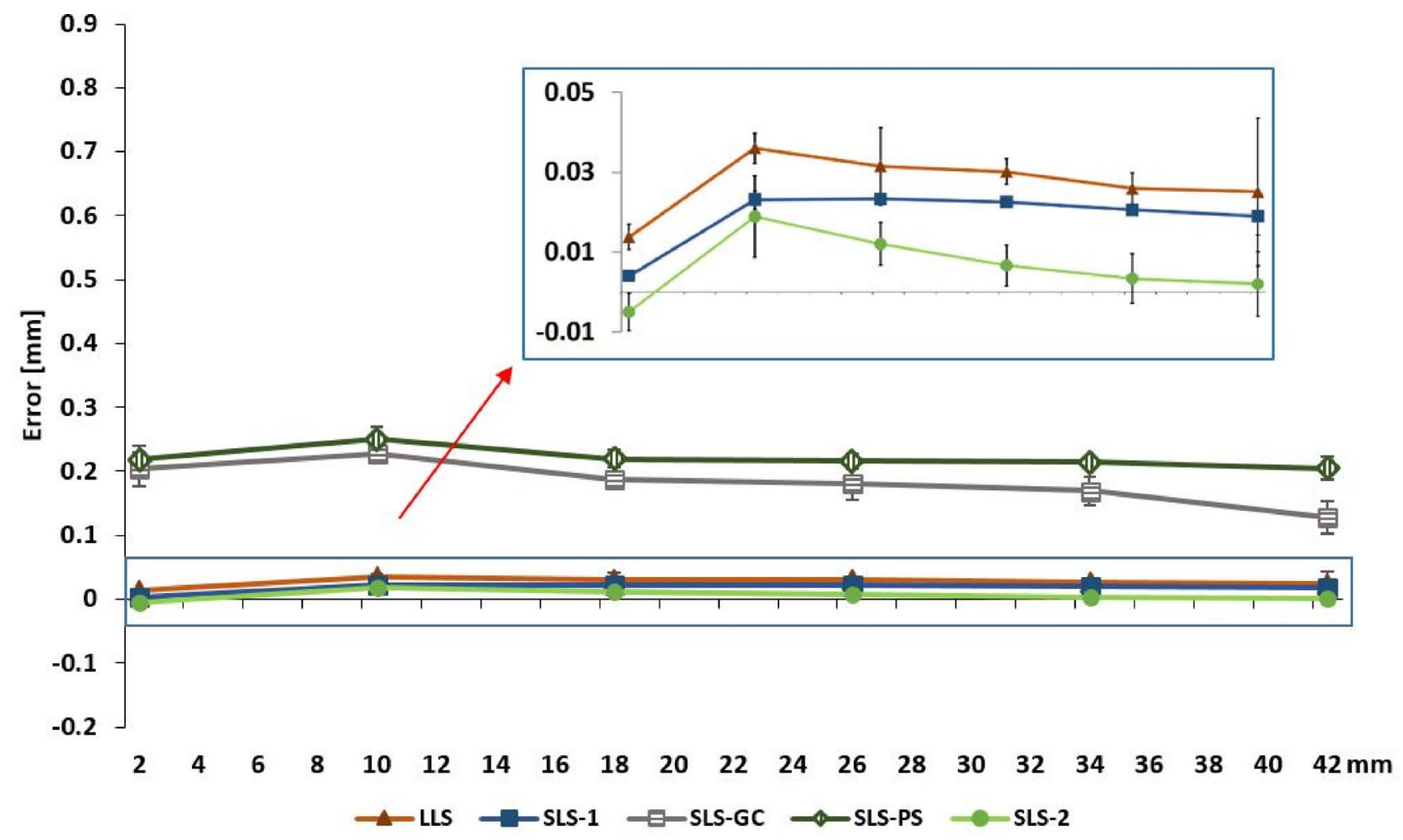

Figure 8 Errors on bidirectional lengths obtained for the ABS-G\#1 step gauge. 
Considering the ABS-G\#1, see Figure 8, the average error computed was on the order of $0.02 \mathrm{~mm}$ for the SLS-1, $0.01 \mathrm{~mm}$ for the SLS-2, while the average error computed for the LLS was about $0.03 \mathrm{~mm}$. Definitely higher were errors registered for the SLS-GC and SLS-PS, on the order of $0.2 \mathrm{~mm}$, with a worse performance for the SLS-PS. The uncertainties were below $0.01 \mathrm{~mm}$ for the SLS-1, SLS-2 and LLS, while they were doubled in the case of SLS-GC and SLS-PS.

The PPS-B\#7, see Figure 9, was characterized by a similar "offset" between the different instruments involved. In particular, errors registered for the SLS-1 were in the same order of the errors obtained for the unidirectional lengths, while the LLS presented errors in the order of $0.01 \mathrm{~mm}$. The PSSRT and the SLS-2 showed a similar trend but with errors which have opposite sign with respect to the other scanners and they were about $-0.01 \mathrm{~mm}$. Finally, SLS-GC and SLS-PS showed errors in the order of 0.5 $\mathrm{mm}$ and $0.6 \mathrm{~mm}$, respectively. Uncertainties were less than $0.01 \mathrm{~mm}$ for the LLS, SLS-1, SLS-2 and PSSRT, while they were about 0.05 and $0.06 \mathrm{~mm}$ for SLS-GC and SLS-PS, respectively.

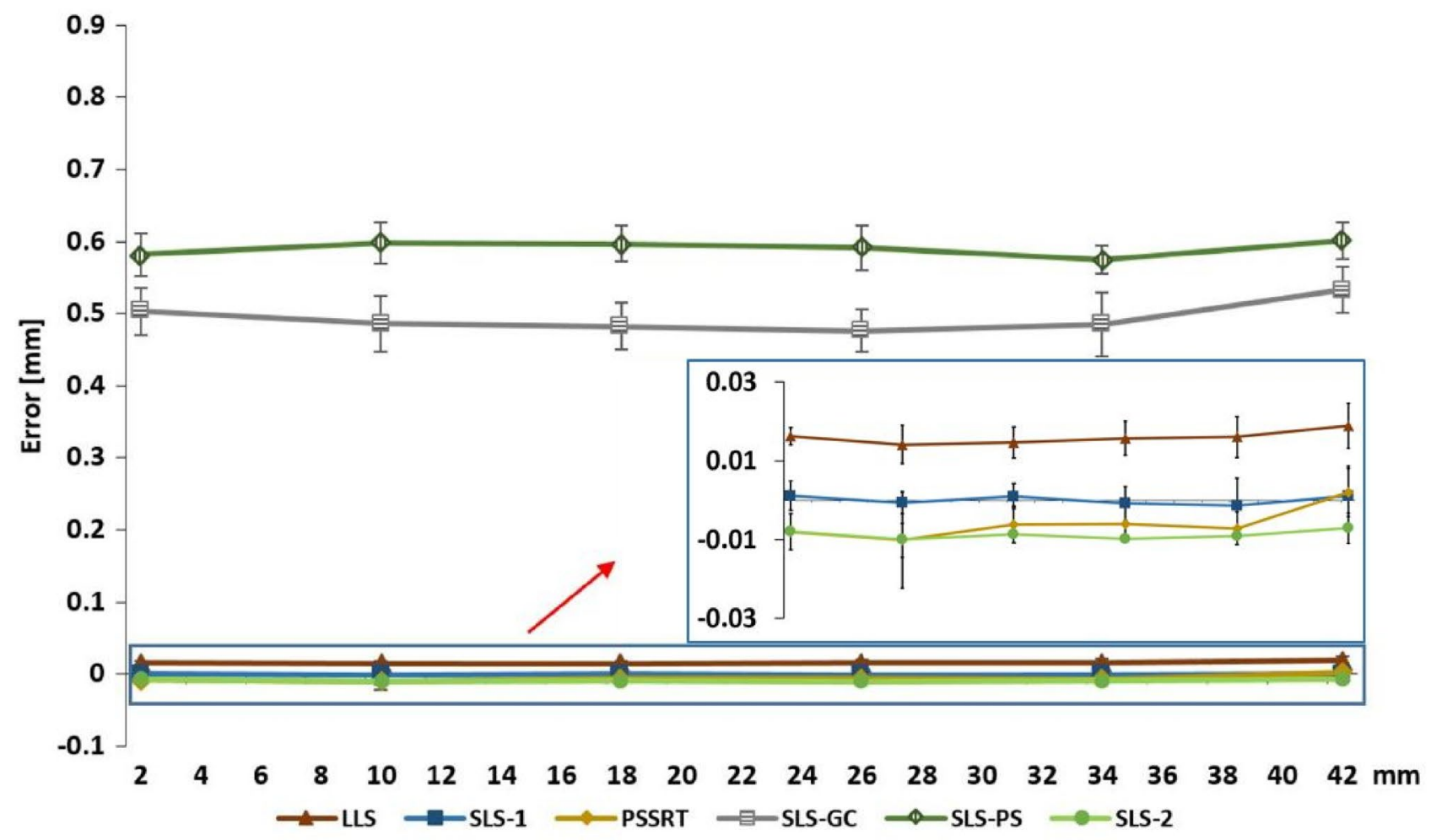

Figure 9 Errors on bidirectional lengths obtained for the PPS-B\#7 step gauge.

The PK-B\#1 was characterized by better defined offsets, see Figure 10. In particular, SLS-2 showed the lowest errors, about $0.02 \mathrm{~mm}$, less than the error registered for SLS-1 and a half of the error registered for the LLS $(0.045 \mathrm{~mm})$. SLS-GC and SLS-PS registered errors on the order of $0.3 \mathrm{~mm}$ and $0.4 \mathrm{~mm}$. Uncertainties were about $0.01 \mathrm{~mm}$ for LLS, SLS-1 and SLS-2, while they were about $0.02 \mathrm{~mm}$ for the SLSGC and SLS-PS. 


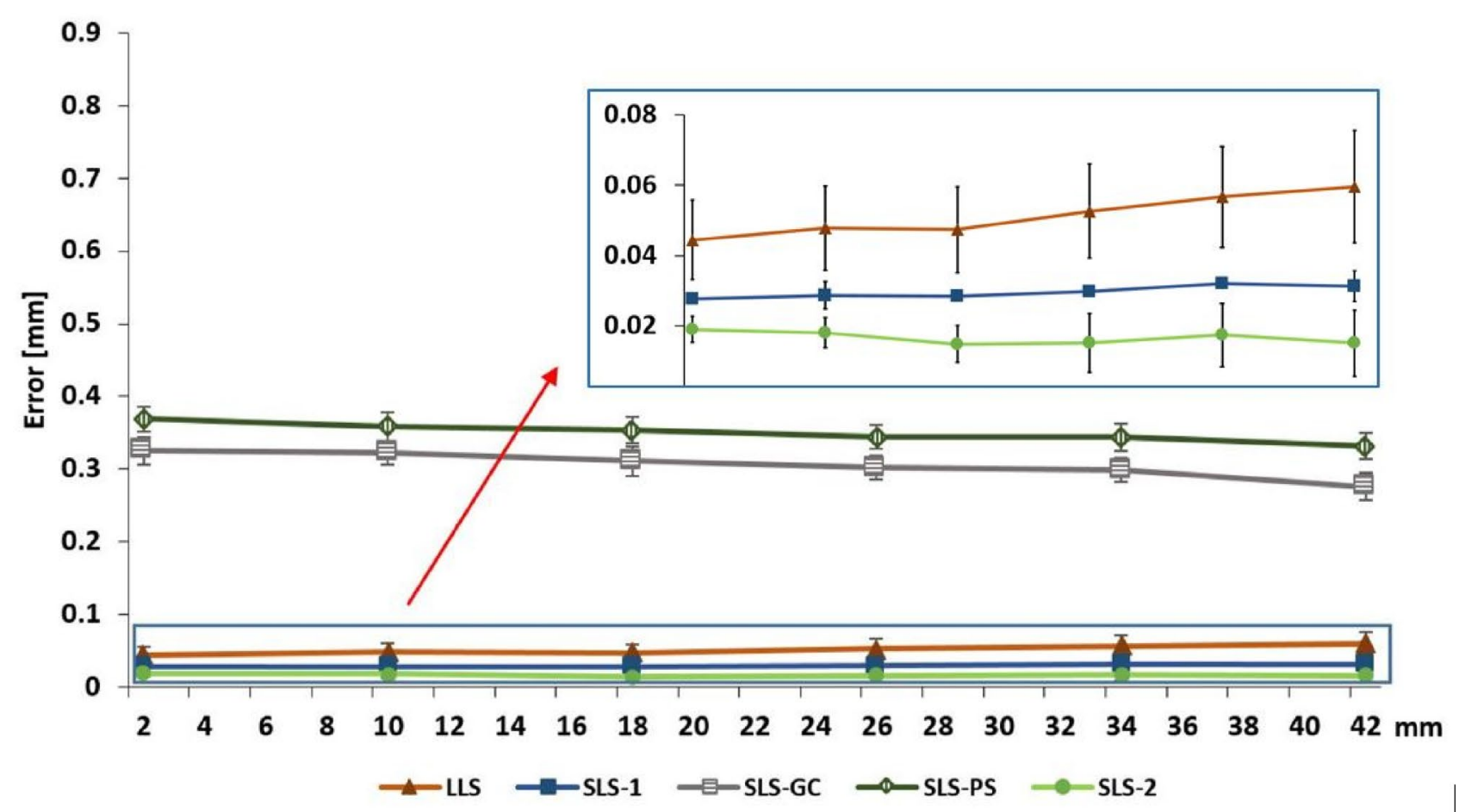

Figure 10 Errors on bidirectional lengths obtained for the PK-B\#1 step gauge.

Finally the two step gauges made of POM were measured and analysed, see Figure 11. The POM-B\#1 was successfully scanned by the LLS and the SLS-GC and SLS-PS. The LLS registered errors on the order of $0.05 \mathrm{~mm}$, SLS-GC, $0.45 \mathrm{~mm}$ and SLS-PS, $0.65 \mathrm{~mm}$. Uncertainties for the two, SLS-GC and SLS-PS, were up to $0.06 \mathrm{~mm}$. Moreover, SLS-PS was the only scanner to produce analysable data of POM-C\#1, which was the worst combination material and colour among the five step gauges, with errors up to $0.8 \mathrm{~mm}$ and uncertainties of $0.08 \mathrm{~mm}$.

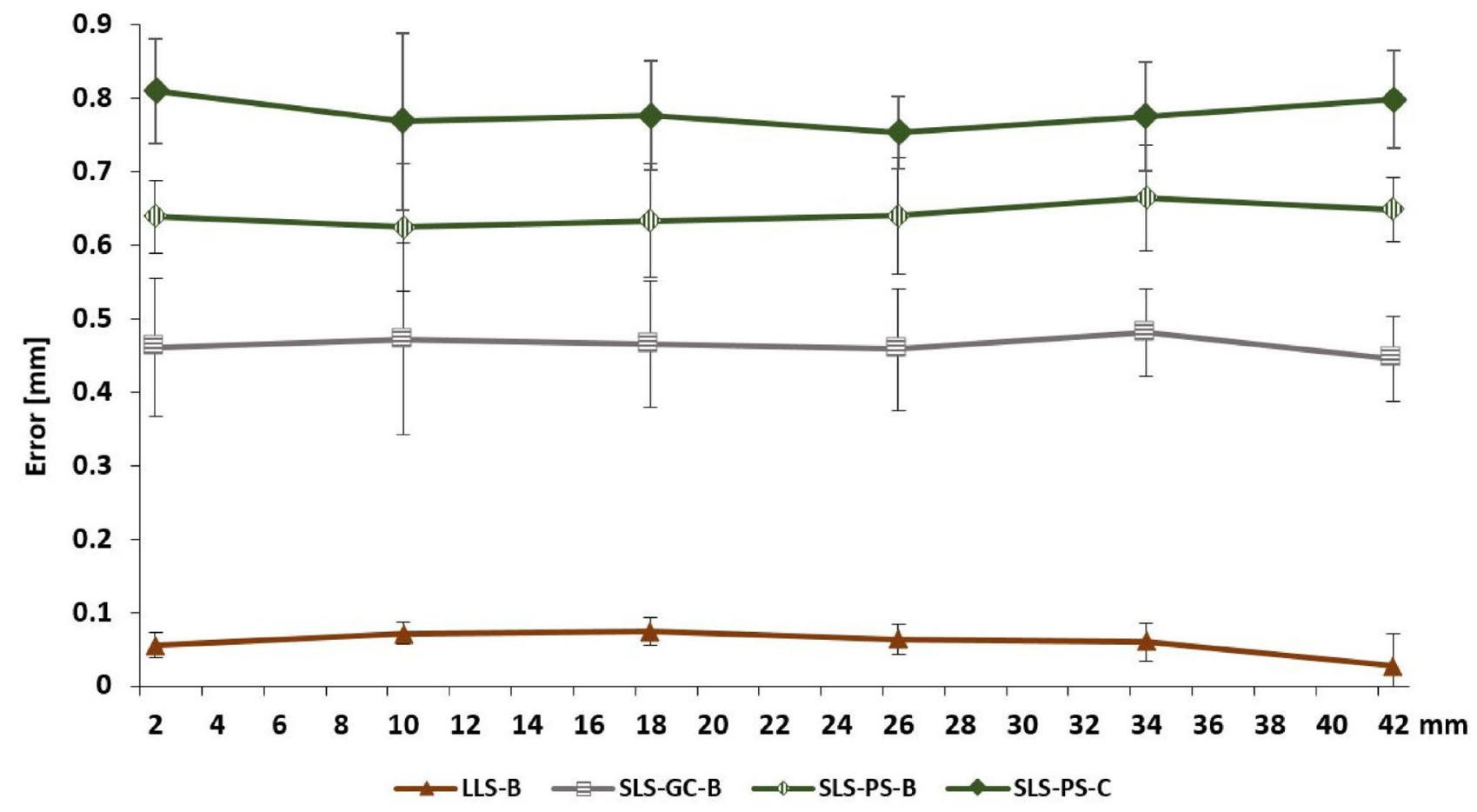

Figure 11 Errors on bidirectional lengths obtained for the POM step gauges. The letter B, after the scanner name, indicates the POM-B\#1 step gauge, while the letter $C$ indicates the POM-C\#1. 


\subsection{Quality of reconstruction: Flatness analysis}

Besides the dimensional verification, flatness registered on the acquired surfaces was considered, in order to take into account the other effect due to the material translucency, which is a different surface quality retrieved, other than a simple bias. This effect leads to an increase of the uncertainty for the cases where a poor reconstruction quality is obtained. A way to evaluate the quality of the reconstruction and, eventually, the differences due to the materials and colours, is the evaluation of the flatness of each groove side, the left groove side and the right groove side, see Figure 12.

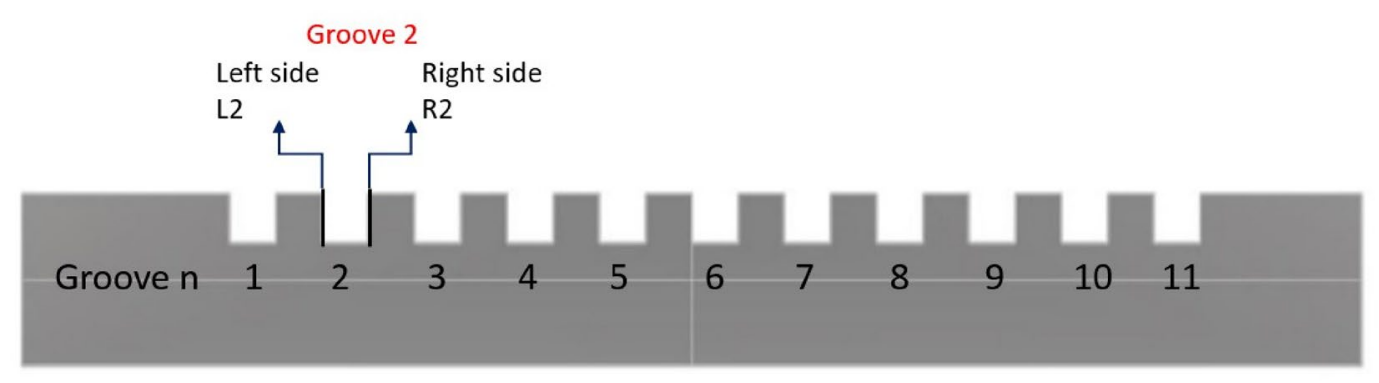

Figure 12 Indication of left and right side of each groove.

The flatness was retrieved with the GOM Inspect software. As it is possible to observe, the flatness values were different according to the instrument used and the material considered. They were about $0.05 \mathrm{~mm}$ for all the scanners involved when used for measuring ABS and PEEK, see Figure 13 and Figure 15, while PPS resulted to be a difficult combination material-colour for the SLS-PS and SLS-GC, see Figure 14, as well as the POM in both its colours, see Figure 16. For the latter, the flatness values were up to $0.15 \mathrm{~mm}$. The analysis showed that the best performance is attributable to the SLS- $1 / 2$ with flatness values on the order of $0.01 \mathrm{~mm}$. Those results are quite in agreement with the dimensional accuracy results.

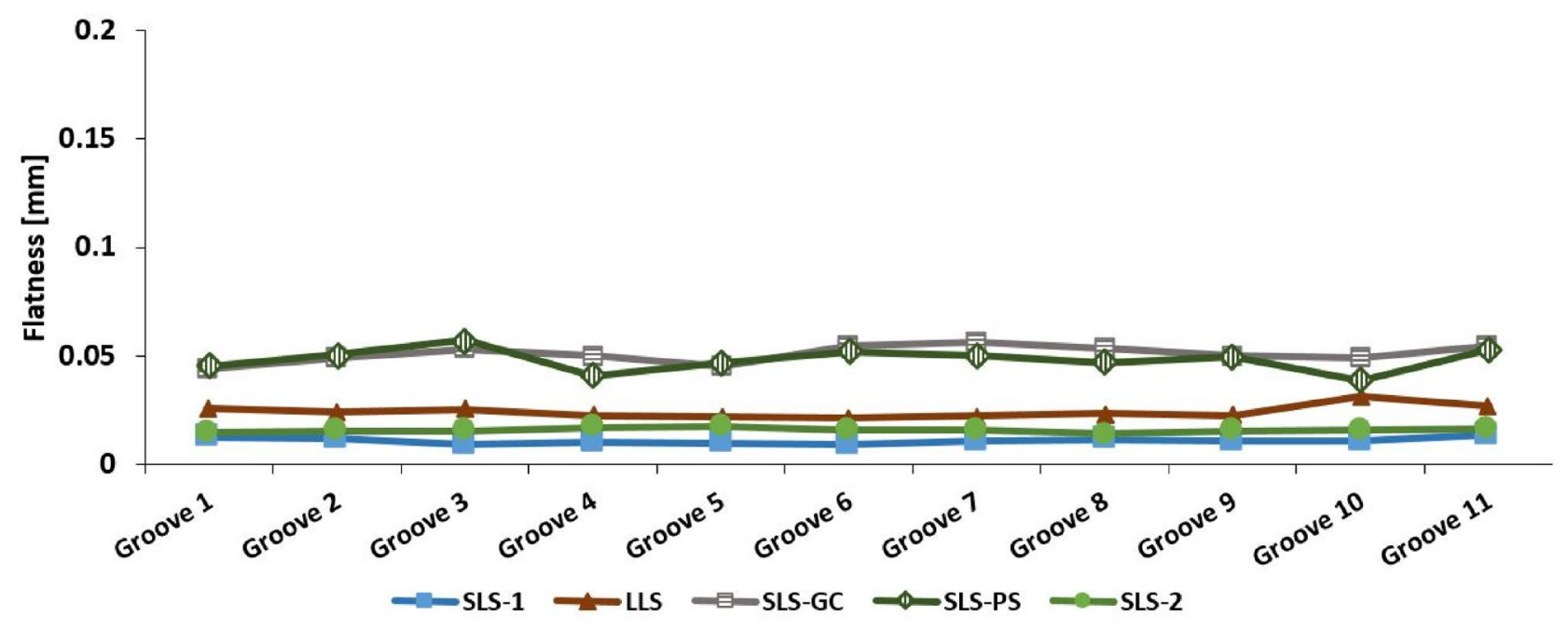

Figure 13 Flatness evaluated on the ABS-G\#1 for each instrument involved. 


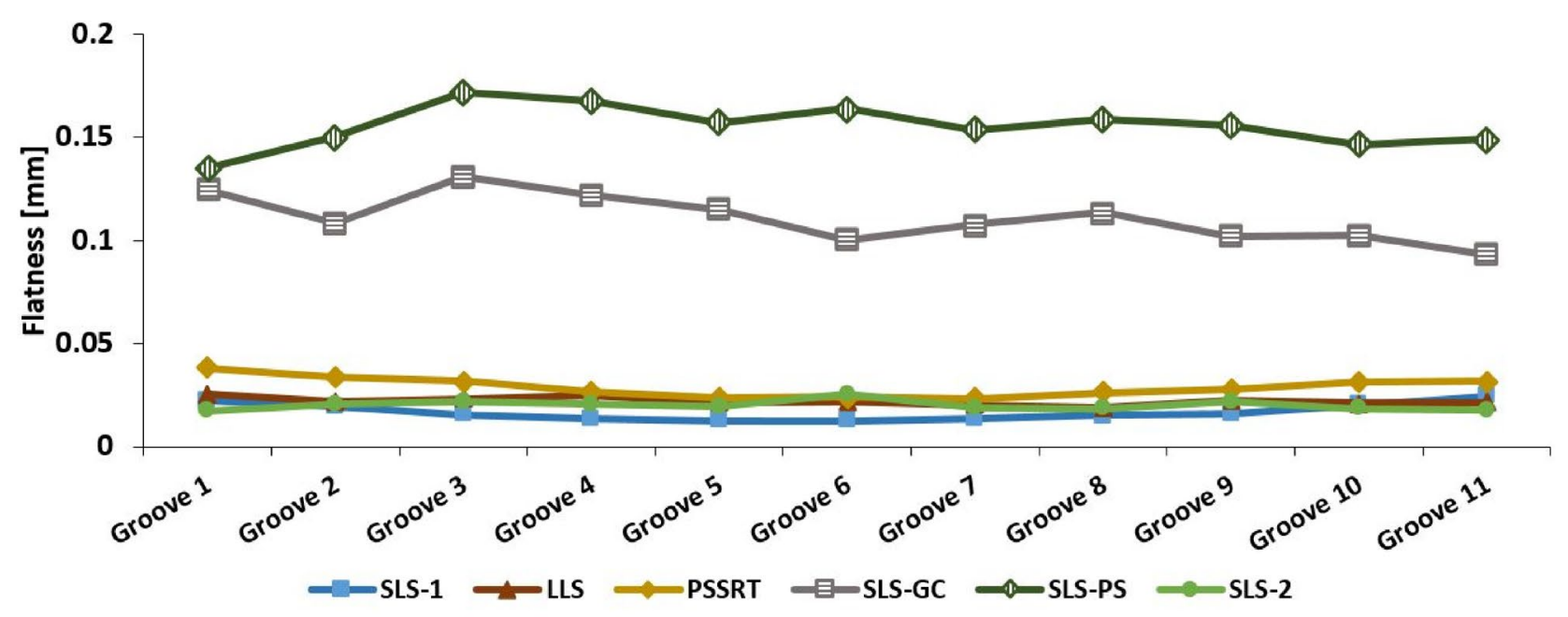

Figure 14 Flatness evaluated on the PPS-B\#7 for each instrument involved.

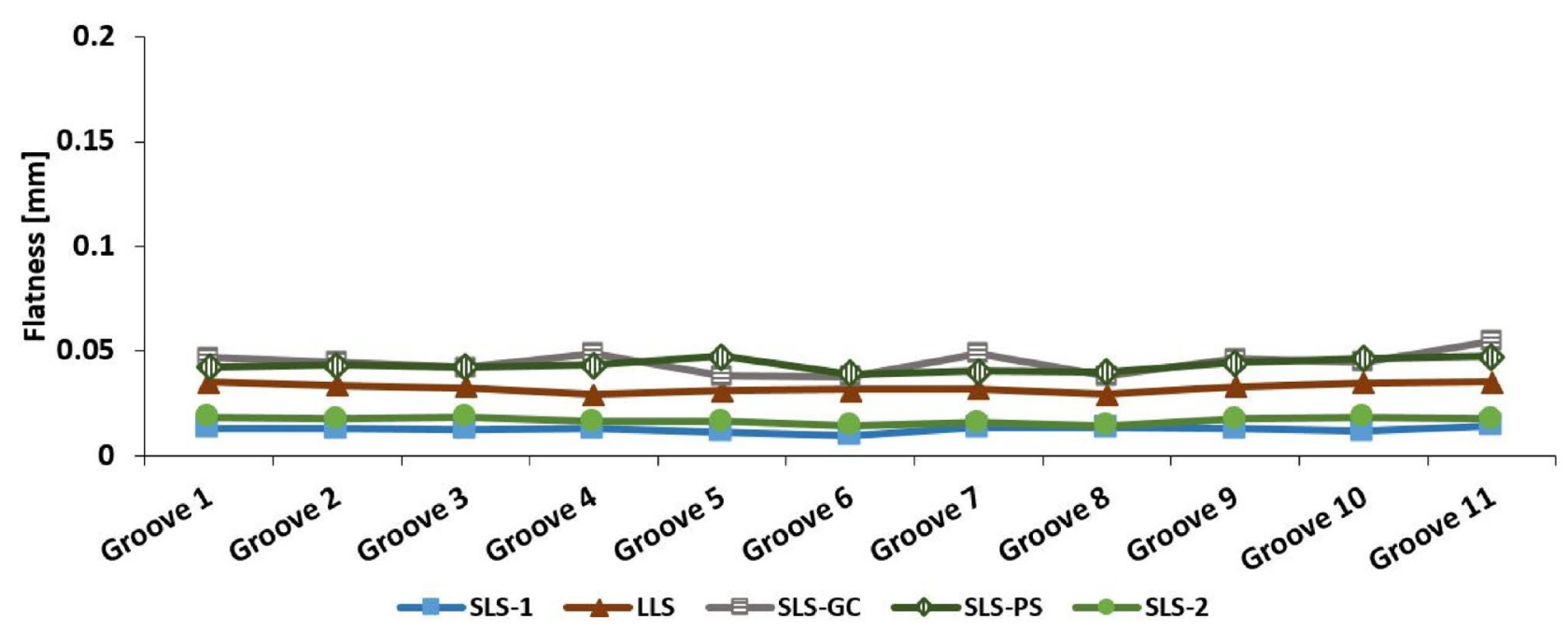

Figure 15 Flatness evaluated on the PK-B\#1 for each instrument involved.

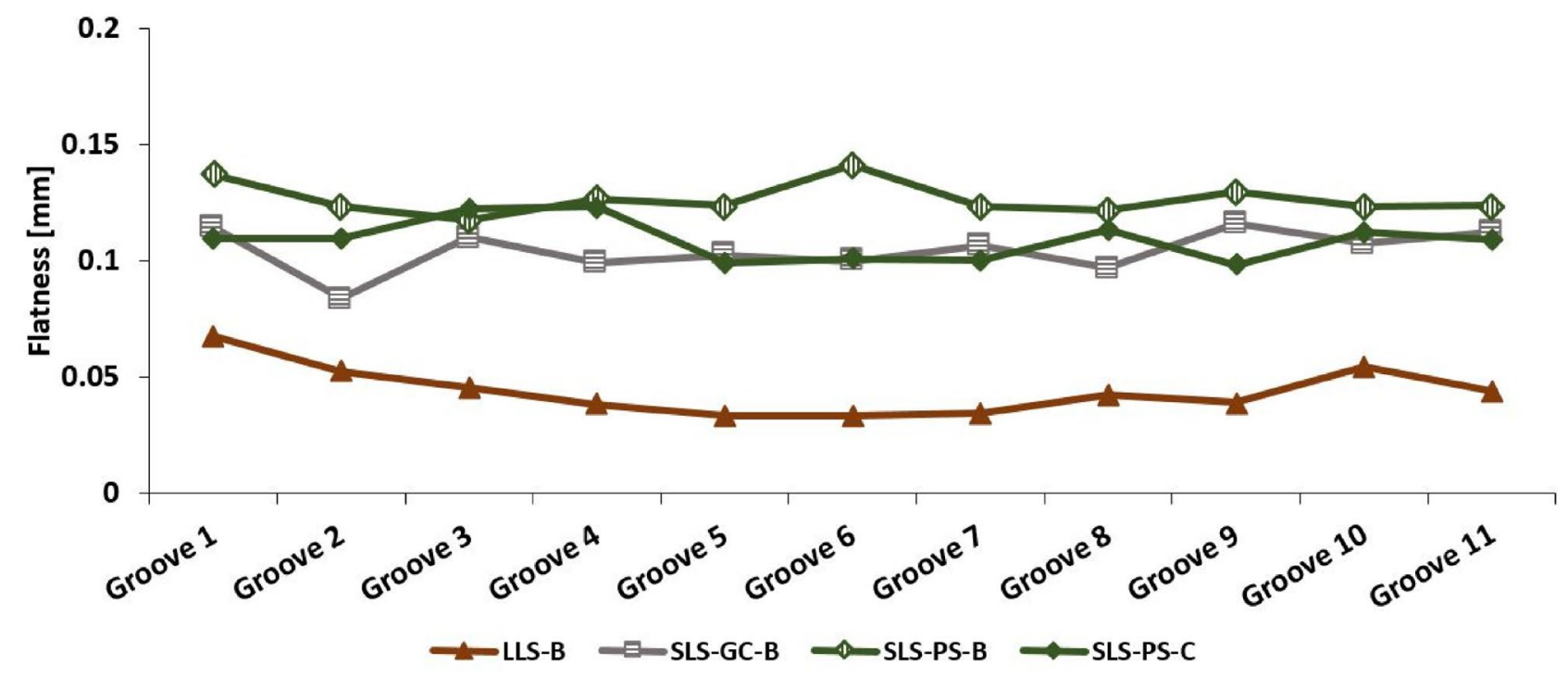

Figure 16 Flatness evaluated on the POM step gauges for each instrument involved. The letter B, after the scanner name, indicates the POM-B\#1 step gauge, while the letter $C$ indicates the POM-C\#1. 


\section{Discussion of results}

The presented measurements are noticeably affected by the interactions between the optical instruments and the material properties of each step gauge. In fact, it is not possible to attribute the significant difference between measured bidirectional lengths to inaccuracies, as all the instruments involved showed similar accuracies, assessed through the unidirectional lengths. The unidirectional errors were found within $\pm 0.005 \mathrm{~mm}$ for the LLS, SLS-1, SLS- 2 and PSSRT, and within $-0.05 \mathrm{~mm}$ and $0.01 \mathrm{~mm}$ for the SLS-GC and SLS-PS. In comparison, the bidirectional errors were found between -0.01 $\mathrm{mm}$ and $0.8 \mathrm{~mm}$, depending on the instrument and the step gauge. There are, indeed, significant differences observed between the optical instruments. Some of them, like the SLS-1/2, are only weakly affected, while others like the SLS-GC/PS are much more strongly affected. To facilitate further analysis and discussion, we introduce the optical penetration depth,

$$
\varepsilon_{i j}=\frac{\text { Error }_{i j}}{2},
$$

where $\varepsilon_{i j}$ is the penetration depth computed for the $i^{\text {th }}$ step gauge with the $j^{\text {th }}$ optical scanner. The Error $_{i j}$ is the average bidirectional error estimated according to Eq. (1.4) considering the error of each measurand, covering the step gauge length. Moreover, an uncertainty was associated to each value and it was evaluated as the standard deviation of the above mentioned bidirectional length errors.

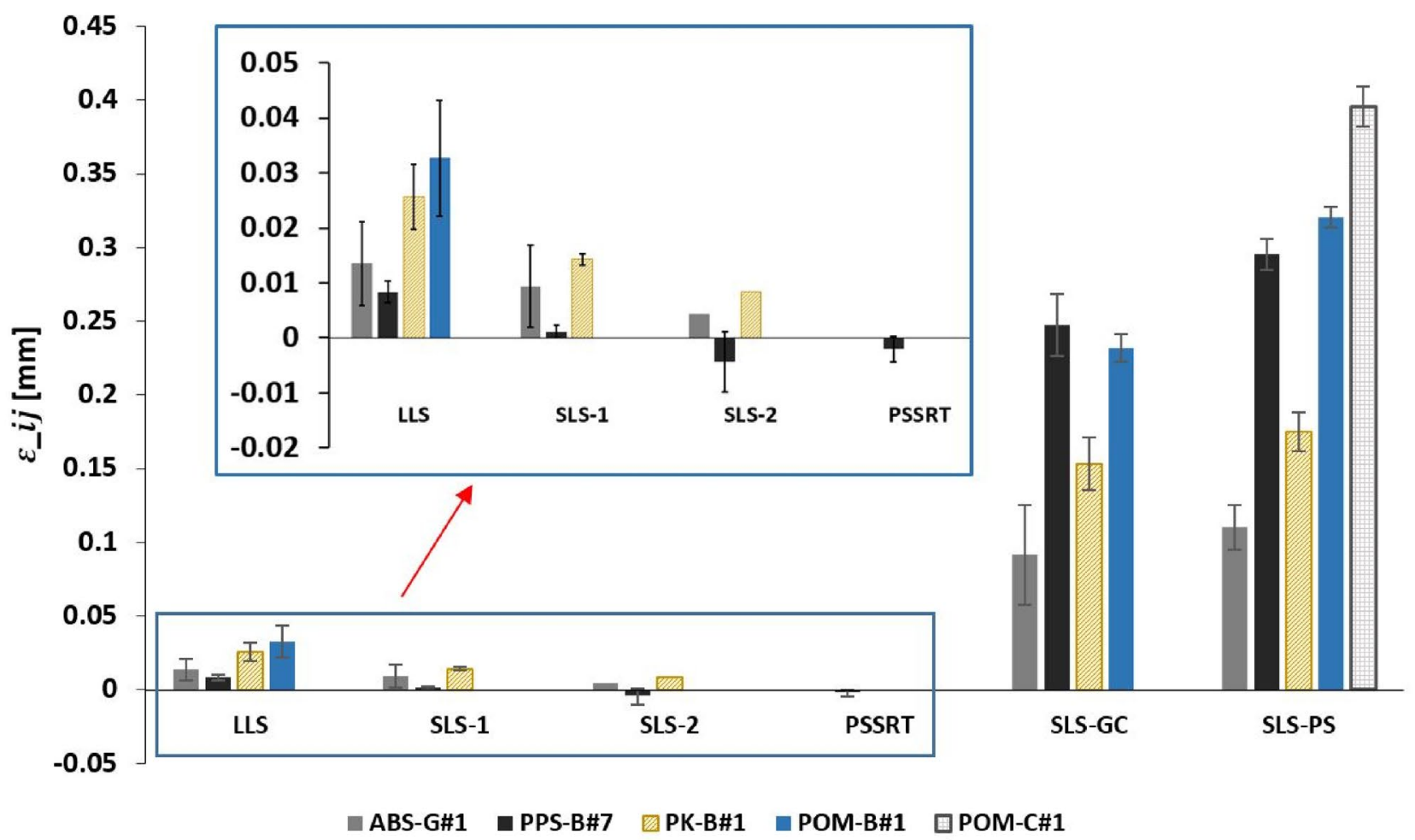

Figure 17 Optical penetration depth for each combination material-scanner.

Looking at Figure 17, the PPS-B\#7 produced the smallest subsurface scattering effect for the LLS, SLS-1, SLS-2 and PSSRT, with values comparable with the errors evaluated on unidirectional lengths. The behaviour is different for the SLS-PS and SLS-GC. Over all, the ABS-G\#1 step gauge displays the smallest values of subsurface scattering for all the scanners involved and only in the case of SLS-GC and SLS-PS it reached the $0.2 \mathrm{~mm}$. The PK-B\#1 registered a more pronounced effect for all the scanners, while the $\mathrm{POM}-\mathrm{B}$ and POM-C were the worst materials to scan. 
On the instrument side, the SLS-GC and SLS-PS were affected the most by the translucency of the materials involved. One possible explanation is the light source, which is different from the others. The scanner SLS-GC/PS uses a red, green and blue (white) light source and, in terms of subsurface scattering, each of the wave lengths must undergo different levels of scattering resulting in chromatic aberrations. As opposed to this, the GOM ATOS scanners (SLS-1/2) use a blue light source, which has been reported to reduce the effects of subsurface scattering [33]. In addition, their method of phase-shifting is applied (sequentially) in two orthogonal directions. In a sense, this could be considered a sequential version of modulated phase-shifting [17]. The LLS scanner uses a different source entirely, namely a red beam laser line. We have found no previous studies reporting advantages of using a red laser beam. However, it is possible to use the intensity differences in the beam shape to get a fairly accurate estimate of the laser entry point [8]. Furthermore, a laser may experience problems due to speckle in the presence of subsurface scattering. The wide bandwidth of the LLS laser is likely to reduce said speckle effects, and use of the beam shape may be the reason why this scanner performs better than SLS-GC and SLS-PS. The improved accuracy of SLS-1/2 as compared with LLS is then likely due to the blue light used in the SLS1/2. The PSSRT scanner, which considers only the PPS-B\#7 step gauge, showed an offset of opposite sign compared with the others. This means that the optical effect in this case seems to be the opposite of the light penetration. In fact, the photogrammetric system used in this case exploits the photogrammetric principle and is a passive system, without the projection of any pattern or a laser beam. As such, it relies on diffuse illumination i.e. light from several directions. The light paths in case of subsurface scattering are then much more intricate than for the other scanners. To fully understand the differences observed in this study, further investigations will have to be carried out considering photogrammetric systems equipped as active systems. We note that the same effect was found for the SLS-2. Both of the SLS1-1/2 and the LLS are commercial systems that may or may not apply a plethora of (unknown) post-processing steps to the measured data, many of which could indeed explain the origin of this negative offset.

Finally, the flatness was also analysed as an index of the quality of reconstruction. Similar to the errors (see Eq. 1.5), the average flatness of all groves for each step gauges is considered, see Figure 18. Generally, results are quite in agreement with the ones regarding the dimensional errors. The most critical material was the POM-B and POM-C. The best materials were the ABS and PEEK, while the PPS severely affected the SLS-GC and SLS-PS measurements, in agreement with the dimensional verification. This behaviour is different from all other scanners involved, and can be explained by the black colour of the material. Black objects are challenging for optical scanners by their very nature of reflecting very little light. This means that factors such as camera quality become more important, and different systems are affected differently. The SLS-GC/PS scanner may very well be less suited for black objects than the others. The lowest registered values of form error were found for the SLS-1 and SLS-2 scanners. Considering all the step gauges they were on the order of $0.02 \mathrm{~mm}$. The quality of reconstruction i.e. the flatness was also included in the uncertainty budgets. The dual effect of the translucency of materials is thus accounted for in both measurement of the dimensional errors (the bias), and the computation of uncertainties of the same dimensional errors. 


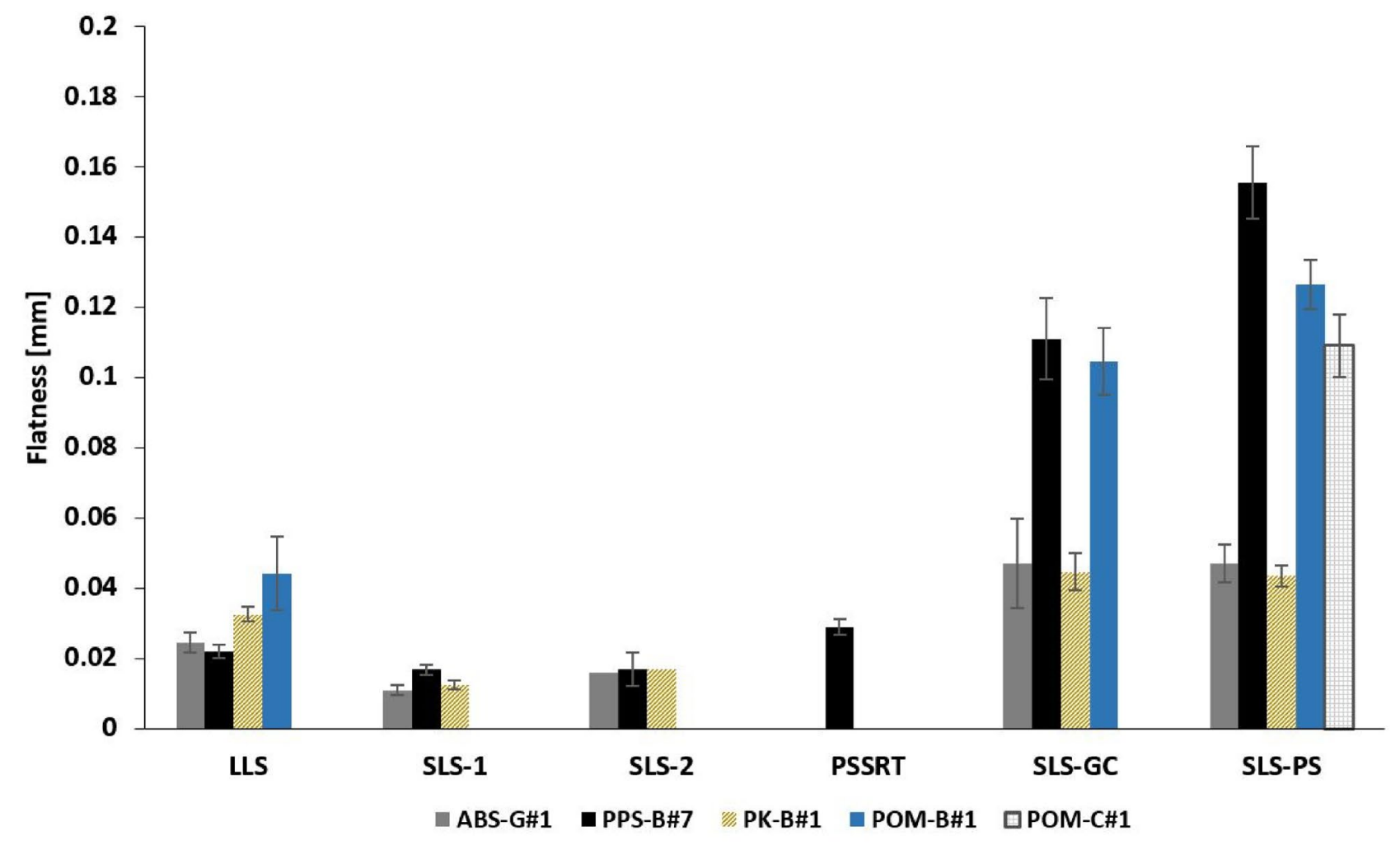

Figure 18 Flatness values for each combination material-scanner.

\section{Conclusion}

In this work, five step gauges made of different materials and colours were scanned and measured with different 3D optical scanners, with the aim to investigate their optical interactions. The dimensional analysis highlighted that the translucent polymer materials have a significant effect on the dimensional verification and its amount depends on the instrument involved. The best behaviour was registered for the SLS-1 and SLS-2, which were the commercial structured light scanners, while the most marked effects due to the translucency of the materials analysed were registered for the SLS-GC and SLS-PS.

The LLS was slightly more sensitive to the subsurface scattering and the PSSRT, which was a passive system, registered a small error below $0.01 \mathrm{~mm}$ with the opposite sign respect to the other instruments. It is important to note, however, that results obtained for the PSSRT refer to only one step gauge, the one made of PPS, and a further investigation is required for the analysis using the PSSRT as an active system. The homebuilt structured light scanner (SLS-GC/PS) displays significantly higher subsurface scattering effect, if compared to the commercial scanners. It was, however, the only scanner able to scan all the supplied translucent objects.

One reason the DTU scanner outperforms the commercial ones (in terms of widest acceptable range of step gauge material) might simply be a low noise tolerance built in to the commercial scanner's software. The DTU scanner is allowed to operate on arbitrarily high tolerances, which are tailored for the experiments in question. While this is also likely to contribute to the relatively large errors observed for the DTU scanner, it does allow it to operate on materials that the other scanner simply cannot. Poor results are sometimes better than no results, and the SLS-GC/PS might be one way towards understanding the influence of subsurface scattering in optical 3D scanning. However, the obtained results suggest problems that need to be addressed before taking steps toward developing new (e.g. bias compensation) techniques for scanning translucent materials. For one, the light source plays an important role, which, upon further studies, should be considered. 
Finally, a quality analysis of the reconstruction was conducted by considering the flatness of each groove side, showing results in good agreement with the dimensional verification. Moreover, the fitting error was considered in the uncertainty budget and this allowed us to observe the dual effect of the optical interaction between the 3D scanner and the translucent materials: the measurement bias and the increased uncertainty.

In conclusion, the subsurface scattering effect is relevant and should be considered when the dimensional verification of polymers has to be carried out. This work represents a first step towards a subsequent bias compensation. The further idea could be to embed the compensation of the bias produced by the optical interactions between the scanner and measured materials in the measuring procedure. To this end, further experiments and studies are needed and more instruments and more materials and colours of interest for the manufacturing field, has to be involved in the analysis.

\section{Bibliography}

[1] Imkamp D, Berthold J, Heizmann M, Kniel K, Peterek M, Schmitt R, et al. Challenges and trends in manufacturing measurement technology - the "Industrie 4.0" concept. J Sensors Sens Syst 2016;83:32535. doi:10.1515/teme-2015-0081.

[2] Hopmann C, Fischer T. New plasticising process for increased precision and reduced residence times in injection moulding of micro parts. CIRP J Manuf Sci Technol 2015;9:51-6. doi:10.1016/j.cirpj.2015.01.004.

[3] Yussef A, Hollister SJ, Dalton PD. Additive manufacturing of polymer melts for implantable medical devices and scaffolds. Biofabrication 2017. doi:10.1088/1758-5090/aa5766.

[4] Guerra MG, Volpone C, Galantucci LM, Percoco G. Photogrammetric measurements of 3D printed microfluidic devices. Addit Manuf 2018;21:53-62. doi:10.1016/j.addma.2018.02.013.

[5] Sims-Waterhouse D, Piano S, Leach R. Verification of micro-scale photogrammetry for smooth threedimensional object measurement. Meas Sci Technol 2017;28:055010. doi:10.1088/1361-6501/aa6364.

[6] Farrell TJ, Patterson MS, Wilson B. A diffusion theory model of spatially resolved, steady-state diffuse reflectance for the noninvasive determination of tissue optical properties in vivo. Med Phys 1992;19:879-88. doi:10.1118/1.596777.

[7] Pickering JW, Prahl SA, van Wieringen N, Beek JF, Sterenborg HJCM, van Gemert MJC. Double-integratingsphere system for measuring the optical properties of tissue. Appl Opt 1993;32:399. doi:10.1364/A0.32.000399.

[8] Abildgaard OHA, Kamran F, Dahl AB, Skytte JL, Nielsen FD, Thomsen CL, et al. Non-Invasive Assessment of Dairy Products Using Spatially Resolved Diffuse Reflectance Spectroscopy. Appl Spectrosc 2015;69:1096105. doi:10.1366/14-07529.

[9] Dury MR, Woodward SD, Brown SB, McCarthy MB. Surface finish and 3D optical scanner measurement performance for precision engineering. Proc - ASPE 2015 Annu Meet 2015:419-23.

[10] Clark J, Robson S. Accuracy of Measurements Made With a Cyrax 2500 Laser Scanner Against Surfaces of Known Colour. Surv Rev 2004;37:626-38. doi:10.1179/sre.2004.37.294.626doi.org/10.1179/sre.2004.37.294.626.

[11] Voisin S, Page DL, Foufou S, Truchetet F, Abidi MA. Color influence on accuracy of 3D scanners based on structured light. Proc SPIE-Machine Vis Appl Ind Insp XIV 2006;6070:72-80. doi:10.1117/12.643448.

[12] Pantone. X-rite - Colour Checker 2019. https://www.xrite.com/categories/calibrationprofiling/colorchecker-classic (accessed March 29, 2019).

[13] Palousek D, Omasta M, Koutny D, Bednar J, Koutecky T, Dokoupil F. Effect of matte coating on 3D optical measurement accuracy. Opt Mater (Amst) 2015;40:1-9. doi:10.1016/j.optmat.2014.11.020.

[14] Gerbino S, Del Giudice DM, Staiano G, Lanzotti A, Martorelli M. On the influence of scanning factors on the 
laser scanner-based 3D inspection process. Int J Adv Manuf Technol 2016;84:1787-99. doi:10.1007/s00170-015-7830-7.

[15] Chen T, Lensch HPA, Fuchs C, Seidel H-P. Polarization and Phase-Shifting for 3D Scanning of Translucent Objects. 2007 IEEE Conf. Comput. Vis. Pattern Recognit., IEEE; 2007, p. 1-8. doi:10.1109/CVPR.2007.383209.

[16] Rojas-Ochoa LF, Lacoste D, Lenke R, Schurtenberger P, Scheffold F. Depolarization of backscattered linearly polarized light. J Opt Soc Am A 2004;21:1799. doi:10.1364/JOSAA.21.001799.

[17] Chen T, Seidel H-P, Lensch HPA. Modulated phase-shifting for 3D scanning. 2008 IEEE Conf. Comput. Vis. Pattern Recognit., IEEE; 2008, p. 1-8. doi:10.1109/CVPR.2008.4587836.

[18] Gupta M, Agrawal A, Veeraraghavan A, Narasimhan SG. A Practical Approach to 3D Scanning in the Presence of Interreflections, Subsurface Scattering and Defocus. Int J Comput Vis 2013;102:33-55. doi:10.1007/s11263-012-0554-3.

[19] Gupta M, Agrawal A, Veeraraghavan A, Narasimhan SG. Structured light 3D scanning in the presence of global illumination. CVPR 2011, IEEE; 2011, p. 713-20. doi:10.1109/CVPR.2011.5995321.

[20] Kobayashi T, Higo T, Yamasaki M, Kobayashi K, Katayama A. Accurate and Practical 3D Measurement for Translucent Objects by Dashed Lines and Complementary Gray Code Projection. 2015 Int. Conf. 3D Vis., IEEE; 2015, p. 189-97. doi:10.1109/3DV.2015.29.

[21] Chiba N, Hashimoto K. 3D measurement by estimating Homogeneous Light Transport (HLT) Matrix. 2017 IEEE Int. Conf. Mechatronics Autom., IEEE; 2017, p. 1763-8. doi:10.1109/ICMA.2017.8016084.

[22] Inoshita C, Mukaigawa Y, Matsushita Y, Yagi Y. Shape from Single Scattering for Translucent Objects, 2012, p. 371-84. doi:10.1007/978-3-642-33709-3_27.

[23] Godin G, Rioux M, Levoy M, Cournoyer L, Blais F. An assessment of laser range measurement on marble surfaces. 5th Conf Opt 3D Meas Tech 2001:8.

[24] Wilm J, Madruga DG, Jensen JN, Gregersen SS, Brix ME, Guerra MG, et al. Effects of subsurface scattering on the accuracy of optical 3D measurements using miniature polymer step gauges. Proc. euspen's 18th Int. Conf. Exhib. Venice, IT, 2018euspen's 18th Int. Conf. Exhib. Venice, IT, 2018, p. 449-50.

[25] Bianco G, Gallo A, Bruno F, Muzzupappa M. A comparative analysis between active and passive techniques for underwater 3D reconstruction of close-range objects. Sensors (Switzerland) 2013;13:11007-31. doi:10.3390/s130811007.

[26] Lavecchia F, Guerra MG, Galantucci LM. The influence of software algorithms on photogrammetric microfeature measurement's uncertainty. Int J Adv Manuf Technol 2017:1-15. doi:10.1007/s00170-017-0786$\mathrm{z}$.

[27] De Chiffre L, Carmignato S, Cantatore A, Jensen JD. Replica calibration artefacts for optical 3D scanning of micro parts. Proc. 9th Int. Conf. Eur. Soc. Precis. Eng. Nanotechnology, EUSPEN, 2009, p. 352-5.

[28] Cantatore A, Angel J, De Chiffre L. Material investigation for manufacturing of reference step gauges for CT scanning verification. Proc. 12th Int. Conf. Eur. Soc. Precis. Eng. Nanotechnology, EUSPEN, 2012, p. 129-32.

[29] Eiríksson ER, Wilm J, Pedersen DB, Aanæs H. Precision and Accuracy Parameters in Structured Light 3-D Scanning. Int Arch Photogramm Remote Sens Spat Inf Sci 2016;XL-5/W8:7-15. doi:10.5194/isprsarchives-XL-5-W8-7-2016.

[30] Lavecchia F, Guerra MG, Galantucci LM. Performance verification of a photogrammetric scanning system for micro-parts using a three-dimensional artifact: adjustment and calibration. Int J Adv Manuf Technol 2018;96:4267-79. doi:10.1007/s00170-018-1806-3.

[31] ISO 14253-2:2011. Geometrical product specifications (GPS) -- Inspection by measurement of workpieces and measuring equipment -- Part 2: Guidance for the estimation of uncertainty in GPS measurement, in calibration of measuring equipment and in product verification 2011.

[32] ISO/IEC Guide 98-3. Uncertainty of measurement - Part 3: Guide to the expression of uncertainty in measurement (GUM:1995) 2008. 
[33] Zhang C, Rosenberger M, Breitbarth A, Notni G. Wavelength dependency of optical 3D measurements at translucent objects using fringe pattern projection. In: Harding KG, Zhang S, editors., 2017, p. 1022007. doi:10.1117/12.2262090. 